Métodos de diferenças finitas para opções americanas

Jose Antonio Solano Atehortúa

\author{
DISSERTAÇÃO APRESENTADA \\ AO INSTITUTO DE MATEMÁTICA E ESTATÍSTICA \\ DA UNIVERSIDADE DE SÃO PAULO \\ PARA OBTENÇÃO \\ DO GRAU DE MESTRE EM CIÊNCIAS
}

Área de Concentração: Matemática Aplicada

Orientador: Prof. Dr. Nelson Mugayar Kuhl

Durante a elaboração deste trabalho o autor recebeu apoio da CAPES

São Paulo, março de 2007. 


\title{
Métodos de diferenças finitas para opções americanas
}

\author{
Este exemplar corresponde à \\ dissertação defendida por \\ Jose Antonio Solano Atehortúa \\ e aprovada pela comissão julgadora.
}

São Paulo, março de 2007.

Banca examinadora:

Prof. Dr. Nelson Mugayar Khul (IME-USP)

Prof. Dr. Gerson Francisco (IFT-UNESP)

Prof. Dr. Oswaldo Luiz do Valle Costa (PTC-USP) 


\section{Agradecimentos}

Ao grupo de pesquisas de São Paulo: Itamonte, Florianopolis, Mar del Plata, Caldera Norte por ter-me concedido ótimas condições para minha iniciação na pesquisa.

Agradeço ao Instituto de Matemática e Estatística da Universidade de São Paulo e em particular ao meu orientador o Prof. Dr. Nelson Mugayar Khul.

Em forma especial a CAMA.

À CAPES, pelo apoio financeiro. 
Uno tiene que seguir haciendo lo que estava haciendo. 


\section{Resumo}

Este trabalho apresenta um estudo de métodos de diferenças finitas para se avaliar uma opção americana sob um ativo-objeto que paga dividendos. A discretização do problema de fronteira livre associado, quando formulado como uma desigualdade variacional, conduz a um problema de complementaridade linear em cada passo de tempo. Os esquemas de diferenças finitas estudados permitem resolver os problemas de complementaridade linear de forma eficiente com o algoritmo de Elliott-Ockendon. Este fato é consequência da estrutura da matriz da parte implícita da discretização, que é uma M-matriz tridiagonal, da consistência da discretização e da condição inicial. Resultados numéricos são apresentados para se validar a teoria. A relevância de esquemas dissipativos é brevemente abordada com uma simulação para o parâmetro DELTA.

Palavras chave. Opção Americana, fronteira livre, método de diferenças finitas, problema de complementaridade linear. 


\section{Abstract}

In this work, finite difference methods for the pricing of american options are studied. The discretization of the associated free boundary problem, when formulated as a variational inequality, leads to a linear complementarity problem to be solved at each time step. The finite difference schemes studied allow to solve these linear complementarity problems efficiently with the Elliott-Ockendon algorithm. This fact follows from the structure of the matrix associated with the implicit part of the discretization, which is a tridiagonal M-matrix, from the consistency of the discretization and from the initial condition. Numerical results are presented to validate the theory. The relevance of dissipative schemes if briefly discussed by a simulation for the DELTA parameter.

Key words. American option, free boundary, finite difference method, linear complementarity problem. 


\section{Sumário}

Introdução 1

1 Teoria Básica de Opções $\quad \mathbf{5}$

1.1 Modelo de Precificação de Opções de Black e Scholes . . . . . 6

1.2 Avaliando Opções Americanas . . . . . . . . . . . . . . . . . . 9

2 Aproximação Numérica e Opções Americanas 14

2.1 Análise de diferenças Finitas . . . . . . . . . . . . . . . 14

2.2 Complementaridade linear . . . . . . . . . . . . . 26

3 Testes computacionais $\quad 42$

3.1 Teste de Convergência . . . . . . . . . . . . . . . . 44

3.2 o delta . . . . . . . . . . . . . . . . 45

4 Conclusões 48

Apêndice: Derivação da equação de Black e Scholes 50

Referências bibliográficas $\quad 55$ 


\section{Introdução}

Nos últimos anos o crescimento dos mercados financeiros tem sido associado com a criação e expansão de novos produtos e serviços, entre os quais os produtos derivados são de primeira ordem. Entre os derivativos mais comuns temos as opções.

Uma opção é um instrumento financeiro que confere a seu titular o direito de comprar ou vender um ativo-objeto a um preço determinado (preço de exercício). Os ativos objeto incluem ações, índices de ações, moedas, instrumentos de dívida, commodities e contratos futuros. Para o lançador da opção (investidor que vende a opção), ao direito do titular se opõe uma obrigação futura, caso esse direito seja exercido pelo titular. Chama-se opção americana aquela que pode ser exercida em qualquer data a partir da emissão até o vencimento, e opção européia aquela que só será exercida na data de vencimento.

O prêmio de uma opção é o preço pelo qual a opção é negociada, cujo valor é determinado pelo preço corrente e volatilidade do ativo-objeto, preço de exercício, prazo de vencimento da opção e pela taxa de juros. O procedimento pelo qual se determina o prêmio de uma opção deve refletir a possibilidade de lucro ou prejuízo futuro do lançador, devido às variações na cotação do ativo-objeto. Uma das questões cruciais da negociação com opções é: o preço da opção é caro ou barato? Essa resposta, juntamente com as expectativas futuras do preço do ativo objeto, determinará a relação ótima de risco e 
Introdução

retorno para a posição tomada.

Uma mudança na matemática financeira foi iniciada pela publicação do trabalho de Fischer Black e Myron Scholes [11]. Nele, os autores mostraram que determinadas opções poderiam ser avaliadas pela solução de uma equação diferencial parcial parabólica regressiva.

De um ponto de vista matemático, é significativo que esta equação possa ser transformada na equação do calor.

Assim, o vasto arsenal de técnicas para resolver analíticamente ou para aproximar numericamente a equação do calor pode ser empregado em neste importante aspecto da modelagem financeira. Quando soluções fechadas não podem ser obtidas, ou quando as fórmulas para a solução exata são complicadas de usar na prática, as soluções numéricas surgem como uma maneira natural de resolver o problema.

Em particular, o presente trabalho centra-se em aplicar os esquemas explícito, implícito, Crank-nicolson, e Crandall-Douglas para a precificação de vanilla stock options ${ }^{1}$ todos eles olhados como caso particular do esquema mais geral conhecido como $\theta$-ponderado [27]. Outros esquemas que nós aplicamos teste computacionais é o BDF2 (second order backward difference formula) e Crandall-Douglas 3 níveis. Esses esquemas são muito populares na comunidade de estudo de transferência de calor. Explorando características especiais desta discretização a serem apresentadas mais adiante, daremos a base para uma metodologia de precificação da opção numa forma extremamente eficiente.

Para opções européias, isto requer a solução de um problema de valor inicial na fronteira. Dado que o operador matricial em cada passo é tridiagonal, pode-se resolver este problema em um tempo que é linear no número

\footnotetext{
${ }^{1}$ As opções aqui descritas são às vezes denominadas de derivativos plain vanilla ou 'padrão'. Alguns derivativos que fogem do padrão são simplesmente carteiras com dois ou mais opções plain vanilla. Os derivativos fora do padrão são chamados de opções exóticas ou, simplesmente, exóticos.
} 
de pontos espaço-temporais da grade. O resultado é uma solução numérica rápida e precisa do problema de precificação de uma opção européia.

Entretanto, se nós permitirmos o exercício adiantado da opção então nós temos de fato um problema de opção americana. Assim, obtemos um problema de fronteira livre. Isto pode ser visto como uma desigualdade variacional obviando assim a necessidade de seguir a fronteira livre. A aproximação é construída fazendo uso de um sistema de coordenadas escolhido para reduzir a equação de Black e Scholes à equação do calor.

O operador de difusão é discretizado com os esquemas BDF2 e com o método $\theta$-ponderado fazendo uso de condições de fronteira de Dirichlet na fronteira artificial. Entretanto, após a discretização obtemos um problema de complementaridade linear (PCL) que tem que ser resolvido em cada passo de tempo. Mostrando que o esquema usado produz uma matriz tridiagonal de Minkowsky (M-matriz) conforme a evolução do tempo, e observando uma propriedade no sinal dos vetores que se geram em aqueles PCLs, nós estamos em condições de resolver estes PCL's pelo algoritmo de Elliott-Ockendon. É de suprema importância para este estudo que o algoritmo de Elliot-Ockendon é executado em tempo linear.

A eficiência desta aproximação é comprovada em opções put e call americanas pagando dividendos.

Vamos agora fazer uma descrição geral do conteúdo, motivações e objetivos desta obra.

No capítulo 1 vamos fazer uma revisão das noções básicas da teoria de opções financeiras. O propósito do capítulo é familiarizar o leitor com certas propriedades e definições básicas, e fundamentalmente fixar uma notação que será utilizada nos capítulos seguintes. Para maiores detalhes pode-se consultar [7].

No capítulo 2 apresenta-se uma base geral para analisar as propriedades de esquemas de discretização em diferenças finitas para resolver as equações 
Introdução

de precificação. Para um estudo mais profundo o leitor pode consultar o trabalho clássico de Richtmeyer e Morton [9]. O capítulo 2 é concluído com descrição de um algoritmo para a discretização em diferenças finitas da formulação em complementaridade diferencial parcial. Discretizando o problema de complementaridade diferencial parcial, reduzimos o problema de precificação de uma opção americana à solução de uma seqüência de problemas de complementaridade linear (LCP).

Existem algoritmos de solução diretos e iterativos para resolver o problema de complementaridade numericamente. Por exemplo, o PSOR é aplicado para precificar opções em [35], um método penalty em [36]. Algoritmos diretos são aplicados em [32] e um método de operador spliting é usado em [37]. O problema de precificar uma opção pode ser formulado também em forma de programação linear. Métodos diretos e programação linear são considerados em [38]. Neste trabalho nós estudamos um método direto nos experimentos numéricos.

Os resultados das experiências computacionais realizadas são apresentadas no capítulo 3, onde ilustram-se as técnicas de diferença finita na solução de exemplos de precificação de opções americanas. Nós discutimos os métodos de grade para resolver as equações de Black-Scholes em termos de:

- aproximação por diferenças finitas.

- condições de fronteira.

- cálculo do valor da opção em um instante de tempo dado.

Os experimentos numéricos mostram que a discretização no tempo por médio dos esquemas BDF2 e Crandall-Douglas 3 níveis possuem melhores propriedades de estabilidade e producen menos oscilações na solução numérica devido a seu caráter de métodos dissipativos.

Finalmente, algumas conclusões são dadas bem como temas de pesquisas futuras. 


\section{Capítulo 1}

\section{Teoria Básica de Opções}

O presente capítulo começa com a formulação matemática do problema de precificação para opções européias baseada na equação de Black e Scholes. Como já foi advertido isto representa um modelo simples para o valor de duas opções básicas, as chamadas opção put e opção call. Resultando em um problema de fronteira de uma equação de difusão. Logo nós introduzimos o problema de fixar o preço da opção americana, para este caso, a equação de Black e Scholes resulta em um problema de valor na fronteira livre. Em seguida, nós discutimos a estrutura do problema de complementaridade linear para a precificação de uma opção americana. Em todos os casos os custos de transacção são ignorados. A tabela fornece um glossario da notação matemática que será utilizada subsequentemente. 


\begin{tabular}{ll}
\hline Símbolo & Significando \\
\hline$S$ & Preço atual da ação. \\
$K$ & Preço de exercício da ação. \\
$T$ & Tempo para o vencimento da opção. \\
$t$ & Período atual. \\
$S_{T}$ & Preço da ação no instante $T$. \\
$r$ & Taxa de juro (continuamente capitalizada) livre de risco \\
& para um investimento que vence no instante $T$. \\
$V$ & Valor de uma opção. \\
$C$ & Valor de uma opção americana de compra de uma ação. \\
$P$ & Valor de uma opção americana de venda de uma ação. \\
$\sigma$ & Volatilidade do preço da ação. \\
$D$ & Taxa de dividendo. \\
$S_{f}$ & Instante de exercício ideal. \\
$\Delta$ & A delta. \\
\hline
\end{tabular}

\section{Notações}

\subsection{Modelo de Precificação de Opções de Black e Scholes}

No apêndice nós damos uma derivação da equação de Black e Scholes que em sua forma básica escreve-se como sendo a solução da equação parabólica: ${ }^{1}$

$$
\left\{\begin{array}{lr}
\frac{\partial V}{\partial t}+\frac{\sigma^{2}}{2} S^{2} \frac{\partial^{2} V}{\partial S^{2}}+(r-D) S \frac{\partial V}{\partial S}-r V=0 & \\
S \in(0, \infty) & t \in(-\infty, T), \quad T>0 \\
V(S, T)=g(S) & S \in(0, \infty) \quad \text { condição final. }
\end{array}\right.
$$

Onde $\sigma, r, D$ são constantes reais. A primeira linha de (1.1) é a equação de Black e Scholes, onde a ação sobre a qual a opção é lançada paga dividendos,

\footnotetext{
${ }^{1} \mathrm{~A}$ condição de contorno é fixada no ponto final do tempo, que não é o caso dos tipos clássicos de equações parabólicas.
} 
nesta forma (1.1) é uma equação de tipo convecção difusão em uma dimensão espacial. Com os termos $s^{j} \partial^{j} V / \partial s^{j}$, pode ser reconhecida uma equação diferencial de Euler que pode ser transformada na equação do calor. Em geral, não é simples obter uma solução explícita à equação parabólica acima.

Como por razões de arbitragem temos também

$$
0 \leq C(S(t), t) \leq S(t)
$$

o preço da call é unicamente determinado pelo problema (1.1) se esse problema possui uma única solução. Black e Scholes não resolveram o problema de Cauchy diretamente, seu método consistiu em transformar (1.1) em forma da equação do calor a qual é bem conhecida na física. Por médio das substituições

$$
\begin{array}{cc}
x=\ln \left(\frac{S}{K}\right) & \tau=\frac{1}{2} \sigma^{2}(T-t) \\
C(S, t)=K v(x, \tau) & k_{1}=\frac{2 r}{\sigma^{2}} \quad k_{2}=\frac{2(r-D)}{\sigma^{2}},
\end{array}
$$

obtemos o seguinte problema equivalente a (1.1):

$$
\left\{\begin{array}{c}
v_{\tau}=v_{x x}+\left(k_{2}-1\right) v_{x}-k_{1} v, \quad \tau>0, x \in \mathbb{R} \\
v(x, 0)=\left(e^{x}-1\right)^{+}, x \in \mathbb{R}
\end{array}\right.
$$

Procurando por uma solução da forma

$$
v(x, \tau)=e^{\alpha x+\beta \tau} u(x, \tau)
$$

com constantes reais $\alpha, \beta$ obtemos a equação diferencial parcial

$$
\beta u+u_{\tau}=\alpha^{2} u+2 \alpha u_{x}+u_{x x}+\left(k_{2}-1\right)\left(\alpha u+u_{x}\right)-k_{1} u .
$$


Com a escolha de

$$
\alpha=-\frac{1}{2}\left(k_{2}-1\right), \quad \beta=-\frac{1}{4}\left(\left(k_{2}-1\right)^{2}+4 k_{1}\right)
$$

obtemos a forma

$$
u_{\tau}=u_{x x} \quad \tau>0, x \in \mathbb{R}
$$

com condição final

$$
u(x, 0)=g(x):=\left(e^{\frac{1}{2}\left(k_{2}+1\right) x}-e^{\frac{1}{2}\left(k_{2}-1\right) x}\right) \quad x \in \mathbb{R}
$$

Da teoria da equação do calor é sabido que a solução de (1.4)-(1.5) é dada por

$$
u(x, \tau)=\frac{1}{2 \pi} \int_{-\infty}^{\infty} g(y) e^{-\frac{(x-y)^{2}}{4 \tau}} d y .
$$

O método para o cálculo dessa integral pode se observar na secção 5.4 de [7]. Posteriormente recuperamos a função original $V(S, t)$ usando

$$
V(S, t)=K^{\frac{k_{2}+1}{2}} S^{\frac{1-k_{2}}{2}} e^{-\frac{1}{8} \sigma^{2}\left(\left(k_{2}-1\right)^{2}+4 k_{1}\right)(T-t)} u\left(\ln \left(\frac{S}{K}\right), \frac{\sigma^{2}}{2}(T-t)\right) .
$$

Para opções européias nós temos uma solução fechada, chamada fórmula de Black-Scholes. Estas são, no caso de uma call,

$$
C(S, t)=S e^{-D(T-t)} N\left(d_{1}\right)-K e^{-r(T-t)} N\left(d_{2}\right)
$$

e para o caso da put tem-se

$$
P(S, t)=K e^{-r(T-t)} N\left(-d_{2}\right)-S e^{-D(T-t)} N\left(-d_{1}\right)
$$

onde

$$
N(z):=\frac{1}{\sqrt{2 \pi}} \int_{-\infty}^{z} e^{-\frac{1}{2}(\xi)^{2}} d \xi
$$


e a integral Gaussiana, e as constantes $d_{1}, d_{2}$ são dados por

$$
\begin{aligned}
d_{1} & :=\frac{\ln \left(\frac{S}{K}\right)+\left(r-D+\frac{\sigma^{2}}{2}\right)(T-t)}{\sigma \sqrt{T-t}} \\
d_{2} & :=\frac{\ln \left(\frac{S}{K}\right)+\left(r-D-\frac{\sigma^{2}}{2}\right)(T-t)}{\sigma \sqrt{T-t}} .
\end{aligned}
$$

\subsection{Avaliando Opções Americanas}

Esta seção deriva a estratégia de valuação para as opções americanas que nós seguimos durante todo o trabalho, chamado problema de complementaridade diferencial parcial (PCDP).

$\mathrm{O}$ valor $V$ de uma opção é dada pelo seguinte sistema de desigualdades, onde $f$ é o payoff:

$$
\begin{gathered}
V \geq f \\
\frac{\partial V}{\partial t}+(r-D) S \frac{\partial V}{\partial S}+\frac{1}{2} \sigma^{2} S^{2} \frac{\partial^{2} V}{\partial S^{2}} \leq r V \\
\left(\frac{\partial V}{\partial t}+(r-D) S \frac{\partial V}{\partial S}+\frac{1}{2} \sigma^{2} S^{2} \frac{\partial^{2} V}{\partial S^{2}}-r V\right)(V-f)=0 \\
V(S, T)=f(S) .
\end{gathered}
$$

Este sistema pode entenderse da seguinte maneira. A primeira desigualdade expressa que em cada instante de tempo o preço da opção não pode ser menor que o seu valor intrínseco. A segunda desigualdade reflete que se o valor da opção cresce mais lentamente do que o valor de um bond livre risco, a opção é exercida. A terceira condição reforça o fato de que se o valor de uma opção está acima do seu valor intrínseco, o preço da opção é descrita pela mesma EDP que descreve a correspondente opção européia. A última condição indica que o valor da opção na data de vencimento iguala seu valor intrínseco. No próximo capítulo detalharemos um método para resolver o sistema (1.7). 
Estratégia para tratar com opções americanas : Opções européias, uma put por exemplo, não permitem o exercício adiantado e também é bem sabido que podem alcançar valores $V_{P}(S, t)<K-S$ para $S$ perto de zero ([15]). Como pode-se ver da equação (1.7), isto não pode acontecer para puts americanas, mas para $S>0$ suficientemente pequeno a igualdade se mantém certa. Como $V$ é contínua e monótona, deve existir um valor $0<S_{f}<K$, para o qual a primeira linha em (1.7) muda de igualdade para desigualdade. $S_{f}$ denota o último ponto para o qual a igualdade se cumpre, i.e. $V_{P}\left(S_{f}, t\right)=K-S$, e depende também de $t$. Inicialmente, este ponto de contato $S_{f}(t)$ é desconhecido mas muito importante de saber porque diz se é oportuno reter ou melhor exercer a opção. No último caso, o ponto correspondente $t_{S}$ é chamado tempo de parada. Se $S_{f}(t)$ é calculado, deve obedecer o seguinte

\section{estratégia para opções americanas}
a call: $S<S_{f}(t)$ : reter
$S \geq S_{f}(t):$ exercer
a put: $S \leq S_{f}(t)$ : exercer
$S>S_{f}(t)$ : reter

Se a $p u t$ for executado, a quantidade ganha $\mathrm{K}$ deve ser investida em um ativo livre de risco com taxa $r$ por o tempo restante $(T-t)$.

Se a call for executado, o estoque comprado $K$ é vendido por $S$ e o lucro $S-K$ deve ser investido em um ativo livre de risco.

Da análise anterior se pode concluir sobre o valor da call e da put americanas, que

$$
\begin{array}{ll|ll}
V_{C}(S, t)>(S-K)^{+} & \text {se } S<S_{f}(t) & V_{P}(S, t)=K-S & \text { se } S \leq S_{f}(t) \\
V_{C}(S, t)=S-K & \text { se } S \geq S_{f}(t) & V_{P}(S, t)>(K-S)^{+} & \text {se } S>S_{f}(t)
\end{array}
$$

Desde que $S_{f}$ não é sabido a priori, resolver para $V$ é chamado um problema de valor na fronteira livre. Para poder calcular a fronteira desconhecida 
$S_{f}$, nós necessitamos condições adicionais. Eventualmente nós temos que adicionar uma condição no ponto de contato para ambos a put e a call:

$$
\frac{\partial V_{C}}{\partial S}\left(S_{f}(t), t\right)=1 \quad \frac{\partial V_{P}}{\partial S}\left(S_{f}(t), t\right)=-1
$$

Resumindo todo aqueles fatos para opções americanas obtém-se o seguinte problema de valor na fronteira livre [15]:

\begin{tabular}{|ll|}
\hline Call americana & \\
para $S<S_{f}(t)$ & $V(S, t)>(S-K)^{+} \mathrm{e}$ \\
& $V_{t}+\frac{1}{2} \sigma^{2} S^{2} V_{t t}+(r-D) S V_{S}-r V=0$ \\
para $S>S_{f}(t)$ & $V(S, t)=(S-K)^{+} \mathrm{e}$ \\
& $V_{t}+\frac{1}{2} \sigma^{2} S^{2} V_{t t}+(r-D) S V_{S}-r V<0$ \\
condição de fronteira & $V(0, t)=0$ \\
& $V\left(S_{f}(t), t\right)=S_{f}(t)-K$ \\
& $V_{S}\left(S_{f}(t), t\right)=1$ \\
& $V(S, T)=(S-K)^{+}$ \\
condição final &
\end{tabular}

\begin{tabular}{|ll|}
\hline Put americana & \\
para $S<S_{f}(t)$ & $V(S, t)=(K-S)^{+} \mathrm{e}$ \\
& $V_{t}+\frac{1}{2} \sigma^{2} S^{2} V_{t t}+(r-D) S V_{S}-r V<0$ \\
para $S>S_{f}(t)$ & $V(S, t)>(S-K)^{+} \mathrm{e}$ \\
& $V_{t}+\frac{1}{2} \sigma^{2} S^{2} V_{t t}+(r-D) S V_{S}-r V=0$ \\
condição de fronteira & $\lim _{S \rightarrow \pm \infty} V(S, t)=0$ \\
& $V\left(S_{f}(t), t\right)=S_{f}(t)-K$ \\
& $V_{S}\left(S_{f}(t), t\right)=-1$ \\
& $V(S, T)=(K-S)^{+}$ \\
condição final &
\end{tabular}

Aplicando as transformações (1.2) da seção anterior acima da desigual- 
dades quando avaliamos opções americanas, obtemos diretamente:

$$
\frac{\partial^{2} u}{\partial x^{2}} \leq \frac{\partial u}{\partial \tau}
$$

Recordando que as condições para o problema de valor na fronteira livre (PVFL) para uma call americana em (1.8) levam sob a transformação a

$$
V_{C}(S, t) \geq(S-K)^{+}=K \max \left\{e^{x}-1,0\right\}
$$

substituindo esta expressão em (1.6) têm-se $0<D<r$

$$
\begin{aligned}
u(x, \tau) \geq & \max \left\{e^{x}-1,0\right\} e^{\frac{1}{2}\left(k_{2}-1\right) x+\frac{1}{4}\left(\left(k_{2}-1\right)^{2}+4 k_{1}\right) \tau} \\
& =e^{\frac{1}{4}\left(\left(k_{2}-1\right)^{2}+4 k_{1}\right) \tau} \max \left\{\left(e^{x}-1\right)\left(e^{\frac{1}{2}\left(k_{2}-1\right) x}\right), 0\right\} \\
& =e^{\frac{1}{4}\left(\left(k_{2}-1\right)^{2}+4 k_{1}\right) \tau} \max \left\{e^{\frac{1}{2}\left(k_{2}+1\right) x}-e^{\frac{1}{2}\left(k_{2}-1\right) x}, 0\right\} \\
& =: g(x, \tau) .
\end{aligned}
$$

A condição final para a call americana, $V(S, T)=(S-K)^{+}$implica uma igualdade na equação acima, daí a condição inicial para $u$ é $u(x, 0)=g(x, 0)$. Para $x \rightarrow \pm \infty$, também temos $u(x, \tau)=g(x, \tau)$.

Com um ajuste da função $g$, esta construção ainda funciona para puts americanas. Assim podemos formular o seguinte problema de complementaridade linear:

$$
\begin{aligned}
& \text { Call: } g(x, \tau):=e^{\frac{1}{4}\left(\left(k_{2}-1\right)^{2}+4 k_{1}\right) \tau} \max \left\{e^{\frac{1}{2}\left(k_{2}+1\right) x}-e^{\frac{1}{2}\left(k_{2}-1\right) x}, 0\right\} \\
& \text { Put: } g(x, \tau):=e^{\frac{1}{4}\left(\left(k_{2}-1\right)^{2}+4 k_{1}\right) \tau} \max \left\{e^{\frac{1}{2}\left(k_{2}-1\right) x}-e^{\frac{1}{2}\left(k_{2}+1\right) x}, 0\right\}
\end{aligned}
$$




$$
(P C L) \begin{cases}\left(\frac{\partial u}{\partial \tau}-\frac{\partial^{2} u}{\partial x^{2}}\right) \geq 0, & (u(x, \tau)-g(x, \tau)) \geq 0 \\ \left(\frac{\partial u}{\partial \tau}-\frac{\partial^{2} u}{\partial x^{2}}\right) \cdot(u(x, \tau)-g(x, \tau))=0 & \\ u(x, 0)=g(x, 0) & 0 \leq \tau \leq \frac{1}{2} \sigma^{2} T \\ u(x, \tau)=g(x, \tau) & \text { para } x \rightarrow \pm \infty\end{cases}
$$

No próximo capítulo daremos um método para a solução do (PCL) anterior.

O resultado de resolver a equação diferencial parcial de Black e Scholes (1.1) diretamente, ou aplicar a transformação e resolver a equação de condução de calor, são os mesmos. Tecnicamente é mais fácil resolver esta última por varias razões: A mais obvia é, que (1.4) possui só dois termos, o termo de difusão e a derivada temporal, ao contrario dos quatro termos em (1.1), o qual resulta em um algoritmo mais simples para as calculações. Uma outra vantagem é a ausência do termo de convecção em (1.4), como consequência obtemos um sistema simétrico para resolver, melhor que um não simétrico. Além disso, o termo convectivo em (1.1) pôde conduzir a falta de estabilidade numérica em nossas aplicações, chamadas spurious oscillations oscilações. Não são causadas pela equação diferencial parcial mesma, mas pelo algoritmo numérico particular que nós usamos para resolver-a. Estas dificuldades não estão presentes na equação do calor. 


\section{Capítulo 2}

\section{Aproximação Numérica e Opções Americanas}

O presente capítulo descreve o método apresentado em [14] para a discretização do problema (1.10) na avaliação de opções americanas, a sua relação com o método apresentado por Huang e Pang em [16], além disso é descrito a implementação considerada no presente trabalho, bem como a análise dos resultados obtidos para os diferentes testes computacionais realizados.

\subsection{Análise de diferenças Finitas}

\section{Discretização ou Problema Aproximado}

Entre a grande quantidade de técnicas numéricas para resolver problemas de valor inicial ou problemas de contorno, os métodos de diferenças finitas são muito utilizados. Estes métodos são derivados do truncamento da série de Taylor, onde é dada uma equação diferencial parcial e as condições iniciais e de contorno são substituídas por um conjunto de equações algebricas que são então resolvidas pelas técnicas existentes. Estes métodos possuem uma 
vantagem importante sob outros métodos pela sua simplicidade na análise e desenvolvimento de códigos computacionais na resolução de problemas com geometrias complexas. Discutiremos diferentes esquemas para derivadas de primeira e segunda ordem, e logo as aplicaremos na resolução numérica de problemas de valores iniciais e de contorno para equações diferenciais parciais de segunda ordem.

A aproximação discreta da derivada parcial contínua produz um erro quando os termos da expansão da serie de Taylor são truncados. Este é conhecido como o erro local de truncamento. A EDP de Black e Scholes (1.1) tem três derivadas contínuas, dois são de primeira ordem e uma é de segunda ordem. Se $f(x, t)$ é uma função arbitraria, podem ser obtidas aproximações de varias derivadas parciais de $f$ usando a definição de derivada parcial:

$$
\begin{gathered}
\frac{\partial f(x, t)}{\partial t}=\frac{f(x, t+\Delta t)-f(x, t)}{\Delta t}+O(\Delta t) \\
\frac{\partial f(x, t)}{\partial t}=\frac{f(x, t)-f(x, t-\Delta t)}{\Delta t}+O(\Delta t) \\
\frac{\partial f(x, t)}{\partial t}=\frac{f(x, t+\Delta t)-f(x, t-\Delta t)}{2 \Delta t}+O\left((\Delta t)^{2}\right) \\
\frac{\partial f(x, t)}{\partial t}=\frac{f\left(x, t+\frac{\Delta t}{2}\right)-f\left(x, t-\frac{\Delta t}{2}\right)}{\Delta t}+O\left((\Delta t)^{2}\right) \\
\frac{\partial^{2} f(x, t)}{\partial x^{2}}=\frac{f(x+\Delta x, t)-2 f(x, t)+f(x-\Delta x, t)}{(\Delta x)^{2}}+O\left((\Delta x)^{2}\right) .
\end{gathered}
$$

Para examinar a eficiência de um esquema numérico de diferenças finitas aspectos como consisência, convergência e estabilidade necessitam ser avaliados: 
Seja $\Omega=\left[x_{\text {inf }}, x_{\text {sup }}\right] \times[0, \tau]$ um domínio no plano $(x, t)$ e considere a malha $\left(x_{i}, t_{j}\right), 0 \leq i \leq n$ e $0 \leq j \leq m$ definida em $\Omega$. Depois de substituir todas as derivadas de uma equação diferencial parcial dada

$$
L u=f, \quad(x, t) \in \Omega,
$$

pelos quocientes de diferenças respectivos, obtemos uma equação de diferenças finitas da forma

$$
D U_{i, j}=f_{i, j}, \quad\left(x_{i}, t_{j}\right) \in \Omega
$$

onde $D$ denota o operador de diferenças. Esta última equação representa a forma discreta da equação dada tal que sua solução $U_{i, j}$ aproxima $u(x, t)$ nos pontos da malha.

Erro local de truncamento: O erro local de truncamento $\epsilon_{i, j}$ é a quantidade pela qual a solução $U_{i, j}$ falha em satisfazer a equação $L u=f$, ou seja,

$$
\epsilon_{i, j}=D u_{i, j}-f_{i, j}
$$

Consistência: A equação diferencial $D U_{i, j}=f_{i, j}$ é dita consistente com a equação diferencial parcial $L u=f$ se

$$
\lim _{h, l \rightarrow 0} \epsilon_{i, j}=0
$$

Erro de discretização: $\mathrm{O}$ erro de discretização $V_{i, j}$ é definido como $V_{i, j}=U_{i, j}-u_{i, j}$, onde $U_{i, j}$ é a solução da equação $D U_{i, j}=f_{i, j}$ e $u_{i, j}$ é a solução da equação diferencial original calculada no ponto $\left(x_{i}, t_{j}\right)$. 
Convergência: O esquema de diferenças é dito convergente se

$$
\lim _{h, l \rightarrow 0}\left|V_{i, j}\right|=\lim _{h, l \rightarrow 0}\left(U_{i, j}-u_{i, j}\right)=0, \quad \text { para }\left(x_{i}, t_{j}\right) \in \Omega
$$

Estabilidade: O conceito de estabilidade de um esquema de diferenças finitas é baseado na propagação do erro $E_{i, 0}=V_{i, 0}-U_{i, 0}$, com o crescimento de $j$, onde $U_{i, 0}$ denota os valores iniciais da equação de diferenças $D U_{i, j}=$ $f_{i, j}$, e $V_{i, 0}$ os valores iniciais obtidos da solução de um sistema de diferenças perturbado.

Para uma equação diferencial parcial com uma solução limitada, o esquema de diferenças é dito estável se os erros $E_{i, j}=V_{i, j}-U_{i, j}$ são uniformemente limitados em $i$ quando $j \rightarrow \infty$, isto é,

$$
\left|E_{i, j}\right|<M, \quad \text { para } j>J
$$

onde $M$ é uma constante positiva e $J$ um inteiro positivo. O teorema da equivalência de Lax, estabelece que a estabilidade de uma solução é condição necessária e suficiente para a convergência de um problema de diferenças finitas que é consistente com um problema de valores de contorno bem posto (vide Stirkwerda [18]).

\section{Método $\theta$}

Intoduzimos uma grade discreta com espaçamento $\Delta \tau=k, \Delta x=h$, onde $\Delta x$ é o espaçamento da grade para o preço $(\log )$ do ativo objeto, e $\Delta \tau$ é o espaçamento para o tempo, e fixamos

$$
u_{n}^{m}=u(n \Delta x, m \Delta \tau)
$$


Todos os esquemas envolvem o parâmetro $\alpha$ que é dado por

$$
\alpha=\frac{\Delta \tau}{(\Delta x)^{2}}
$$

Nós aplicamos um esquema de diferenças finitas para o problema de complementaridade linear (1.10). Para a derivada parcial com respecto ao tempo usamos a seguinte aproximação:

$$
\left.\frac{\partial u}{\partial \tau}=\frac{u_{n}^{m+1}-u_{n}^{m}}{\Delta \tau}+O(\Delta \tau)\right)
$$

e para a derivada parcial segunda con respecto da variavel $x$ usamos uma aproximação por diferenças centradas $\theta$-ponderada:

$\frac{\partial^{2} u}{\partial x^{2}}=\theta\left(\frac{u_{n-1}^{m+1}-2 u_{n}^{m+1}+u_{n+1}^{m+1}}{(\Delta x)^{2}}\right)+(1-\theta)\left(\frac{u_{n-1}^{m}-2 u_{n}^{m}+u_{n+1}^{m}}{(\Delta x)^{2}}\right)+O\left((\Delta x)^{2}\right)$, onde $0 \leq \theta \leq 1$. O método $\theta$ é uma generalização dos métodos de diferença finitas por meio do qual, é possível convertir o método a qualquer um de explícito $(\theta=0)$, implicito $(\theta=1)$, Crank-Nicolson $(\theta=1 / 2)$ ou CrandallDouglas dependendo da escolha de $\theta$.

Agora, fazendo uso da nossa aproximação por diferenças finitas obtemos

$$
\frac{\partial u}{\partial \tau}-\frac{\partial^{2} u}{\partial x^{2}} \approx \frac{u_{n}^{m+1}-u_{n}^{m}}{\Delta \tau}-\theta\left(\frac{u_{n-1}^{m+1}-2 u_{n}^{m+1}+u_{n+1}^{m+1}}{(\Delta x)^{2}}\right)-(1-\theta)\left(\frac{u_{n-1}^{m}-2 u_{n}^{m}+u_{n+1}^{m}}{(\Delta x)^{2}}\right)
$$

Assim o método aplicado à equação $u_{t}-u_{x x}=0$ pode-se expandir da seguinte forma

$$
(1+2 \alpha \theta) u_{n}^{m+1}-\alpha \theta\left(u_{n-1}^{m+1}+u_{n+1}^{m+1}\right)=(1-2 \alpha(1-\theta)) u_{n}^{m}+\alpha(1-\theta)\left(u_{n-1}^{m}+u_{n+1}^{m}\right)
$$

- Explicito

$$
u_{n}^{m+1}=(1-2 \alpha) u_{n}^{m}+\alpha\left(u_{n-1}^{m}+u_{n+1}^{m}\right)
$$


- Completamente Implícito

$$
(1+2 \alpha) u_{n}^{m+1}-\alpha\left(u_{n-1}^{m+1}+u_{n+1}^{m+1}\right)=u_{n}^{m}
$$

- Crank-Nicolson

$$
(1+\alpha) u_{n}^{m+1}-\frac{\alpha}{2}\left(u_{n-1}^{m+1}+u_{n+1}^{m+1}\right)=(1-\alpha) u_{n}^{m}+\frac{\alpha}{2}\left(u_{n-1}^{m}+u_{n+1}^{m}\right)
$$

- Douglas (2-níveis no tempo)

$$
\begin{aligned}
& (10+12 \alpha) u_{n}^{m+1}+(1-6 \alpha)\left(u_{n-1}^{m+1}+u_{n+1}^{m+1}\right)=(10-12 \alpha) u_{n}^{m}+(1+6 \alpha)\left(u_{n-1}^{m}+u_{n+1}^{m}\right) \\
& \text { basta tomar } \theta=\frac{1}{2}-\frac{1}{12 \alpha}
\end{aligned}
$$

Apresentamos também mais dois métodos os quais não fazem parte da familia do método $\theta$ :

- Douglas (3-níveis no tempo)

$$
\begin{array}{r}
\left(\frac{1}{8}-\alpha\right)\left(u_{n-1}^{m+1}+u_{n+1}^{m+1}\right)+\left(\frac{5}{4}+2 \alpha\right) u_{n}^{m+1} \\
=\frac{1}{6}\left(u_{n-1}^{m}+u_{n+1}^{m}+10 u_{n}^{m}\right) \\
-\frac{1}{24}\left(u_{n-1}^{m-1}+u_{n+1}^{m-1}+10 u_{n}^{m-1}\right)
\end{array}
$$

- Second-order backward difference (BDF2)

$$
\left(\frac{1}{\Delta t} I+\frac{2}{3} B\right) u^{m+1}=\frac{1}{3 \Delta t} I u^{m-1}-\frac{4}{3 \Delta t} I u^{m}
$$

Onde $B$ é a matriz resultante da discretização no espaço. 


\section{Estabilidade do Método $\theta$}

Se nossos dados numéricos tiverem que arredondarse, e tendo que todas as representações numéricas em um computador são aproximadas, nós não desejamos que os erros que são implícitos em tais representações venham dominarem completamente a resposta numérica do computador. Com um esquema instável, é possível pôr os mesmos algoritmos sobre computadores diferentes, ou usar sistemas de software diferentes no computador, e obter respostas totalmente diferentes.

Existem vários métodos para a análise da estabilidade como por exemplo examinar o número de condições da matriz nos métodos onde se requer da inversão de uma matriz, ou examinar os eigenvalores da matriz que é a multiplicadora do sistema, ou ainda introduzindo uma pequena perturbação no dado inicial.

A análise seguida aqui é como sugerida em ([13]) e ([14]).

Nós supomos infinita precisão aritmética, que temos uma solução $u_{n}^{m}$ da nossa equação de diferenças (2.2), e que a solução perturbada $v_{n}^{m}=u_{n}^{m}+E_{n}^{m}$ , onde $E_{n}^{0}$ representa um erro inicial pequeno e em geral $E_{n}^{m}$ representa o erro no passo de tempo $m$, satisfaz também à mesma equação de diferenças

$$
(1+2 \alpha \theta) E_{n}^{m+1}-\alpha \theta\left(E_{n-1}^{m+1}+E_{n+1}^{m+1}\right)=(1-2 \alpha(1-\theta)) E_{n}^{m}+\alpha(1-\theta)\left(E_{n-1}^{m}+E_{n+1}^{m}\right) .
$$

Nós consideramos as soluções desta equação de diferenças para uma perturbação da forma

$$
E_{n}^{m}=\lambda^{m} \sin (n \omega)
$$

Isto significa que $E_{n}^{0}=\sin (n \omega)$ é uma perturbação pequena harmônica do dado inicial. As possibilidades detalhadas para $\omega$ não são consideradas aqui. Nós estamos interessados nos valores possíveis para o fator de amplificação 
$\lambda$. A visão padrão é que se

$$
|\lambda|>1
$$

então o sistema é instável, e para o sistema ser estável precisamos

$$
|\lambda| \leq 1
$$

Substituindo $E_{n}^{m+1}$ e $E_{n}^{m}$ em (2.3) obtemos:

$$
\begin{aligned}
& \lambda[(1+2 \alpha \theta) \sin (n \omega)-\alpha \theta(\sin ((n-1) \omega)+\sin ((n+1) \omega))] \\
& =[(1-2 \alpha(1-\theta)) \sin (n \omega)+\alpha(1-\theta)(\sin ((n-1) \omega)+\sin ((n+1) \omega))]
\end{aligned}
$$

usando a identidade $\sin ((n+1) \omega))+\sin ((n-1) \omega)=2 \sin (n \omega) \cos (\omega)$ obtemos que:

$$
\lambda(1+2 \alpha \theta-2 \alpha \theta \cos (\omega))=1-2 \alpha(1-\theta)+2 \alpha(1-\theta) \cos (\omega)
$$

Agora nós recordamos a identidade $\cos (\omega)=1-2 \sin ^{2}\left(\frac{\omega}{2}\right)$ para chegar no seguinte valor do fator de amplificação $\lambda$

$$
\lambda=\frac{1-(1-\theta)\left(4 \alpha \sin ^{2}\left(\frac{\omega}{2}\right)\right)}{1+\theta\left(4 \alpha \sin ^{2}\left(\frac{\omega}{2}\right)\right)}
$$

Com o objeto de simplificar a análise dos diversos casos, é conveniente fazer uma substituição. Fixamos

$$
y=4 \alpha \sin ^{2}\left(\frac{\omega}{2}\right)
$$

De modo que considerado em função de $y$ :

$$
\lambda(y)=\frac{1-(1-\theta) y}{1+\theta y} .
$$


Pode-se ver que se $0<\theta<\frac{1}{2}$, então $|\lambda| \leq 1$ só no caso que

$$
0<\alpha \leq \frac{1}{2(1-2 \theta)}
$$

Entretanto, quando $\frac{1}{2} \leq \theta \leq 1$, vemos que $|\lambda| \leq 1 \forall \alpha>0$.

A tabela 2.1 mostra o resumo dos diferentes valores de $\theta$. A Parte Principal do erro de truncamento para cada método é mostrada na coluna PPET, da mesma forma o intervalo de estabilidade deduzido anteriormente pela análise de Von Neumann é mostrado na coluna AVN. Dado que a equação de difusão (1.4) é linear e as aproximações por diferenças finitas que estamos utilizando são consistentes, uma vez provado que um determinado esquema é estável resta usar o teorema de equivalência de Lax para ver se o mesmo convergirá.

\begin{tabular}{llll}
\hline Esquema & $\theta$ & PPET & AVN \\
\hline \hline Explicito & 0 & $O[k]+O\left[h^{2}\right]$ & $\alpha \leq \frac{1}{2}$ \\
Completamente implícito & 1 & $O[k]+O\left[h^{2}\right]$ & OK \\
Crank-Nicolson & $\frac{1}{2}$ & $O\left[k^{2}\right]+O\left[h^{2}\right]$ & OK \\
Crandall-Douglas & $\frac{1}{2}-\frac{1}{12 \alpha}$ & $O\left[k^{2}\right]+O\left[h^{4}\right]$ & OK \\
Crandall-Douglas 3N & NA & $O\left[k^{2}\right]+O\left[h^{4}\right]$ & OK \\
\hline
\end{tabular}

Tabela 2.1: Sumário de propriedades. Onde $k=\Delta \tau$ e $h=\Delta x$.

\section{Teste 1}

Considera-se o caso diffusivo puro com dados iniciais lisos. Black-Scholes com dados iniciais não-lisos tem complicações adicionais. Consideramos a equação de difusão

$$
\frac{\partial u}{\partial \tau}=\frac{\partial^{2} u}{\partial x^{2}}
$$


na região

$$
-2 \leq x \leq 2, \quad \tau \geq 0
$$

com a condição inicial

$$
u(x, 0)=\sin \left(\frac{\pi x}{2}\right)
$$

e as condições de fronteira

$$
u(2, \tau)=u(-2, \tau)=0
$$

Este problema tem a seguinte solução exata

$$
u(x, \tau)=\sin \left(\frac{\pi x}{2}\right) e^{-\frac{\pi^{2} \tau}{4}} .
$$

A tabela 2.2 apresenta os resultados obtidos para a função diferença, ou seja, a diferença entre a solução numérica e a solução analítica no instante $\tau=$ $0.1, M$ representa o número de passos no tempo, $N$ o número de subintervalos em que foi dividido o domínio espacial e $|\cdot|$ é a norma do supremo, $|a|=$ $\max \left|a_{i}\right|, \forall a \in \mathbb{R}^{n}$.

\begin{tabular}{llll}
\hline Método & $N$ & $M$ & Máximo |erro \\
\hline \hline Explicito & 160 & 400 & $3.46952153 E-5$ \\
Completamente implícito & 160 & 400 & $8.41998593 E-5$ \\
Crank-Nicolson & 160 & 40 & $2.41634090 E-5$ \\
Crandall-Douglas & 160 & 40 & $6.09400896 E-7$ \\
Crandall-Douglas 3N & 160 & 40 & $2.37184583 E-6$ \\
BDF & 160 & 40 & $2.24015889 E-5$ \\
Crandall-Douglas & 80 & 10 & $9.75118485 E-6$ \\
Crandall-Douglas 3N & 80 & 10 & $3.43883937 E-5$ \\
BDF & 80 & 10 & $6.47174427 E-5$ \\
\hline
\end{tabular}

Tabela 2.2: Teste 1, $\tau=0.1$ 


\section{Teste 2}

Precificação de uma Opção Européia de compra usando a equação do calor $u_{t}=u_{x x}$, no domínio $x_{\text {inf }} \leq x \leq x_{\text {sup }}$ e $\tau \leq 0.098$, e com os seguintes parâmetros escolhidos $r=0.1, \sigma=0.2, K=1, D=0, x_{\text {inf }}=-6.7$, $x_{\text {sup }}=1.6, n=64, \Delta \tau=0.0049$. Logo $\Delta x=0.13, \alpha=\frac{\Delta \tau}{(\Delta x)^{2}}=0.29$.

No gráfico 2.1 é apresentada a solução exata junto com o payoff para esta opção, a qual possui solução exata dada pela fórmula de Black e Scholes

$$
\left.C(S, t)\right|_{t=0}=S e^{-D(T-t)} N\left(d_{1}\right)-K e^{-r(T-t)} N\left(d_{2}\right)
$$

Aqui $T-t=\frac{2}{\sigma^{2}} \tau$, ou seja $T-t=4.9$. Analogamente, para o preço do ativo subjacente $S$ temos a relação $S=K e^{x}$. quando $x=-6.7$ temos $S=e^{-6.7}=$ 0,001230912 , e para $x=1.6$ temos que o valor de $S$ é $S=e^{1.6}=4,953032424$ As constantes $d_{1}, d_{2}=d_{1}-\sigma \sqrt{T-t}$ para este caso são dados por

$$
\left.d_{1}\right|_{t=0}=\left.\frac{\ln \left(\frac{S}{K}\right)+\left(r-D+\frac{\sigma^{2}}{2}\right)(T-t)}{\sigma \sqrt{T-t}}\right|_{t=0}=\frac{\ln (S)+(0.1+0.02) 4.9}{0.2 \sqrt{4.9}}
$$

Para a resolução numérica vamos resolver primeiramente para uma put e, depois achamos o preço da call por meio da paridade put-call ${ }^{1}$.

A tabela 2.3 apresenta os resultados obtidos após de 20 iterações , $N$ é o número de subintervalos em que foi dividido o domínio espacial e $|\cdot|=$ $\max \left|a_{i}\right|$ para qualquer $a \in \mathbb{R}^{n}$.

\section{Observações}

- O erro para o esquema implícito é maior que aquele cometido pelo esquema explícito, como se previa com a análise do erro de truncamento. Porém, na hora de comparar deve-se levar em conta que o

\footnotetext{
${ }^{1}$ Este procedimento só é possível no caso de opções européias
} 


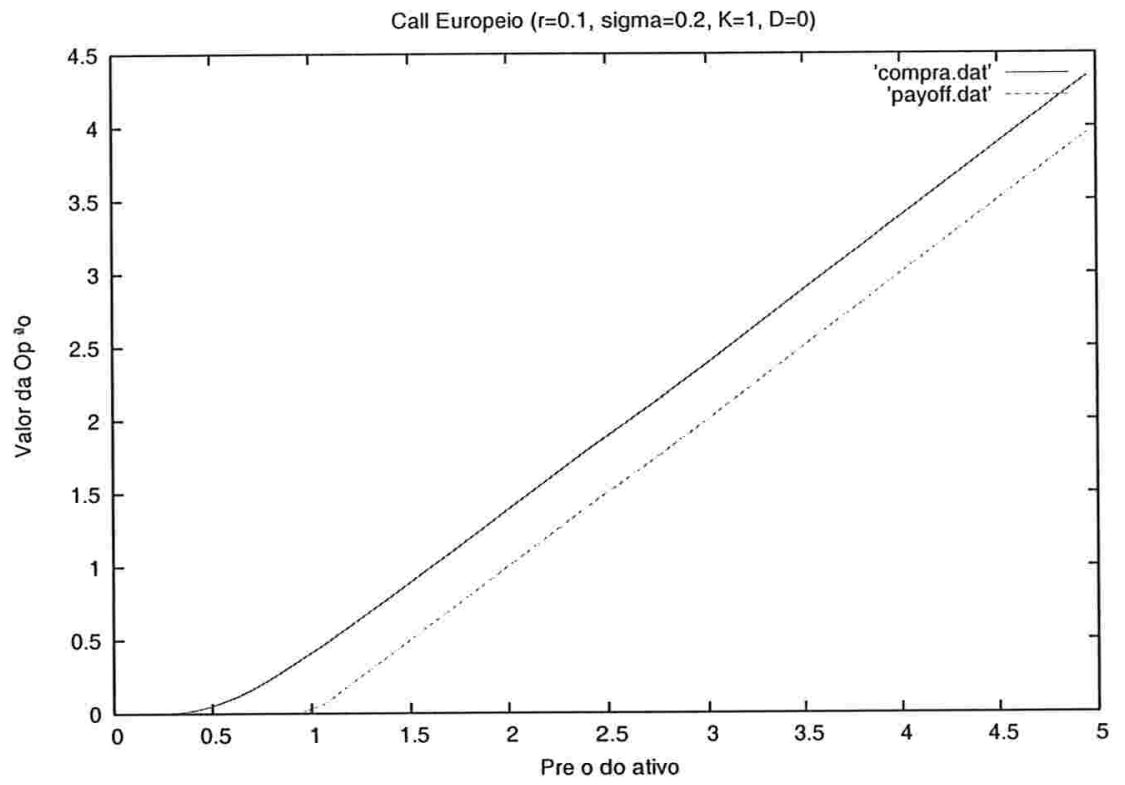

Figura 2.1: Gráfico da solução exata em $\tau=0.090$ do preço de uma opção call européia do teste 2 calculada pelo esquema de Crandall-Douglas, com $\alpha=0.29$ e $\Delta x=0.13$.

esquema implícito permite uma redução em $\Delta x$, quando fixamos $\Delta \tau$, sem que apareça instabilidade o qual não pode ser visto para o esquema explícito.

- Olhando para o esquema de Crank-Nicolson e BDF, observa-se uma melhora significativa sobre o esquema implícito sem que isto carregue custo computacional adicional.

- O esquema de Crandall-Douglas têm resultados superiores sem incremento do custo computacional.

- A tabela 2.3 também indica que se reduzimos o número de pontos espaciais da grade por um fator de $\frac{1}{2}$, o esquema de Crandall-Douglas ainda têm um desempenho melhor que todos os outros esquemas. Só com $\frac{1}{4}$ dos pontos da grade, a precisão produzida é comparável à dos 


\begin{tabular}{lll}
\hline Método & $N$ & $\mid$ erro $\mid$ \\
\hline \hline Explícito & 64 & $1.58322261 E-2$ \\
Completamente implícito & 64 & $5.77881219 E-2$ \\
Crank-Nicolson & 64 & $2.12687753 E-2$ \\
Crandall-Douglas & 64 & $2.89806340 E-4$ \\
Crandall-Douglas 3N & 64 & $8.82841537 E-4$ \\
BDF & 64 & $2.20273079 E-2$ \\
\hline Crandall-Douglas & 32 & $2.28318270 E-3$ \\
Crandall-Douglas 3N & 32 & $2.08766190 E-3$ \\
BDF & 32 & $8.05142016 E-2$ \\
\hline Crandall-Douglas & 16 & $3.95309028 E-2$ \\
Crandall-Douglas 3N & 16 & $3.87266356 E-2$ \\
BDF & 16 & 0.2817100514 \\
\hline
\end{tabular}

Tabela 2.3: Teste 2, $\tau=0.098, N$ representa o número de subdivisões no espaço e || é a norma do supremo.

esquemas de Crank-Nicolson e BDF.

\subsection{Complementaridade linear}

Nesta seção discutiremos a formulação introduzida no capítulo 1 para opções americanas. Por meio da discretização do PCDP (sistema 1.7), nós reduzimos o problema de precificação de uma opção americana para a solução de uma seqüência de problemas de complementaridade linear (PCL).

Nós recordamos o valor de uma opção americana em forma compacta

$$
\begin{gathered}
\left(\frac{\partial u}{\partial \tau}-\frac{\partial^{2} u}{\partial x^{2}}\right) \geq 0, \quad(u(x, \tau)-g(x, \tau)) \geq 0 \\
\left(\frac{\partial u}{\partial \tau}-\frac{\partial^{2} u}{\partial x^{2}}\right) \cdot(u(x, \tau)-g(x, \tau))=0
\end{gathered}
$$


onde $g(x, \tau)$ é a função payoff dada por

$$
g(x, \tau)=e^{\frac{1}{4}\left(\left(k_{2}+1\right)^{2}+4 k_{1}\right) \tau} \max \left(e^{\frac{1}{2}\left(k_{2}-1\right) x}-e^{\frac{1}{2}\left(k_{2}+1\right) x}, 0\right) .
$$

Para uma put, e para uma call é dado por

$$
g(x, \tau)=e^{\frac{1}{4}\left(\left(k_{2}+1\right)^{2}+4 k_{1}\right) \tau} \max \left(e^{\frac{1}{2}\left(k_{2}+1\right) x}-e^{\frac{1}{2}\left(k_{2}-1\right) x}, 0\right)
$$

onde $k_{1}=\frac{2 r}{\sigma^{2}}, k_{2}=\frac{2(r-D)}{\sigma^{2}}$. As condições inicial e de fronteira tornam-se

$$
\begin{gathered}
u(x, 0)=g(x, 0) \\
u(x, \tau) \text { é contínua } \\
\frac{\partial u}{\partial x}(x, \tau) \quad \text { é tão contínua como } g(x, \tau) \\
\lim _{x \rightarrow \pm \infty} u(x, \tau)=\lim _{x \rightarrow \pm \infty} g(x, \tau) .
\end{gathered}
$$

Como já foi notado, uma das principais vantagens da formulação em complementaridade linear, é que não há referencia explícita nenhuma da fronteira livre. Podemos resolver o PCL e posteriormente encontrar a fronteira livre pela condição que o define, a saber

$$
u\left(x_{f}(\tau), \tau\right)=g\left(x_{f}(\tau), \tau\right), \quad \text { e } \quad u(x, \tau)>g(x, \tau) \quad \text { para } \quad x>x_{f}(\tau)
$$

para a put, e para a call têm-se

$$
u\left(x_{f}(\tau), \tau\right)=g\left(x_{f}(\tau), \tau\right), \quad \text { e } \quad u(x, \tau)>g(x, \tau) \text { para } \quad x<x_{f}(\tau) .
$$

Dada uma matriz $A \in \mathbb{R}^{n \times n}$ e um vetor $q \in \mathbb{R}^{n}$, o PCL consiste em achar o 
vetor $z$ que satisfaz as seguintes condições:

$$
\begin{gathered}
A z+q \geq 0 \\
z \geq 0 \\
(A z+q)^{T} \cdot z=0 .
\end{gathered}
$$

Acostuma-se denotar este PCL pelo símbolo $(q, A)$. O nome é por causa da terceira condição, chamada condição de complementaridade a qual requer que pelo menos uma variável do par $(A z+q)_{i}, z_{i}$ deve ser igual a zero na solução do problema, para cada $i=1$ até $n$.

Com o intuito de ilustrar a ligação que a formulação em PCDP (1.10) do problema de precificação de opções têm com o PCL, suponha o seguinte PCDP:

$$
\begin{aligned}
& u(x, \tau) \geq g(x, \tau) \\
& u_{\tau}-u_{x x} \geq 0 \\
& \left(u_{\tau}-u_{x x}\right)(u-g)=0 \\
& 0 \leq \tau \leq \frac{\sigma^{2}}{2} T, \quad-\infty \leq x \leq \infty \\
& u(x, 0)=g(x, 0)
\end{aligned}
$$

Este sistema pode ser resolvido numericamente com um esquema de diferenças finitas, para a solução aproximada temos que truncar o domínio de modo que $x$ se encontre entre $x_{\text {inf }}$, e $x_{\text {sup }}$. Por exemplo, usando o método $\theta$ sobre uma grade regular com tamanho de passo $\Delta x=\frac{x_{\text {sup }}-x_{\text {inf }}}{n}$ no espaço e um tamanho de passo temporal de $\Delta \tau=\frac{\sigma^{2}}{2} \frac{T}{M}$. A cada passo do tempo $m, u$ é aproximado pelo vetor $\left(u_{0}^{m}, u_{1}^{m}, \ldots, u_{n}^{m}\right)$, onde $u_{i}^{m}=u\left(x_{i}, m \Delta \tau\right)$, $x_{i}:=x_{0}+i \Delta x, i=0, \ldots, n$, e $x_{0}=x_{i n f}$. Assim a função paoyff $g$ é aproximada por $g_{n}^{m}=g(n \Delta x, m \Delta \tau)$ 
O sistema (2.4) acima é então aproximado por

$$
\begin{gathered}
u_{n}^{m+1} \geq g_{n}^{m+1} \text { para } m \geq 1 \\
(1+2 \alpha \theta) u_{n}^{m+1}-\alpha \theta\left(u_{n-1}^{m+1}+u_{n+1}^{m+1}\right) \geq b_{n}^{m} \\
\left(-\alpha \theta u_{n-1}^{m+1}+(1+2 \alpha \theta) u_{n}^{m+1}-\alpha \theta u_{n+1}^{m+1}-b_{n}^{m}\right) \cdot\left(u_{n}^{m+1}-g_{n}^{m+1}\right)=0 .
\end{gathered}
$$

Onde como usual $\alpha$ é definido por (2.1) e o vetor $b_{n}^{m}$ é igual a:

$$
\begin{gathered}
b_{1}^{m}=u_{1}^{m}+\alpha(1-\theta)\left(g_{0}^{m}-2 u_{1}^{m}+u_{2}^{m}\right)+\alpha \theta g_{0}^{m+1} \\
b_{n}^{m}=(1-2 \alpha(1-\theta)) u_{n}^{m}+\alpha(1-\theta)\left(u_{n-1}^{m}+u_{n+1}^{m}\right) \quad n=2, \ldots, N-2 \\
b_{N-1}^{m}=u_{n-1}^{m}+\alpha(1-\theta)\left(u_{n-2}^{m}-2 u_{n-1}^{m}+g_{n}^{m}\right)+\alpha \theta g_{n}^{m+1} .
\end{gathered}
$$

Fazendo uma simples mudança de variáveis $z_{i}:=u_{i}-g_{i}$, este sistema é equivalente ao PCL $(q, A)$, onde $A$ é uma matriz $(N-1) \times(N-1)$ e $q$ é um vetor $(N-1)$ definidos por

$$
\begin{gathered}
A=\left[\begin{array}{cccccc}
1+2 \alpha \theta & -\alpha \theta & 0 & 0 & \ldots & 0 \\
-\alpha \theta & 1+2 \alpha \theta & -\alpha \theta & 0 & \ldots & 0 \\
0 & -\alpha \theta & 1+2 \alpha & -\alpha \theta & \ldots & 0 \\
\vdots & & \ddots & \ddots & \ddots & \vdots \\
0 & \ldots & 0 & -\alpha \theta & 1+2 \alpha \theta & -\alpha \theta \\
0 & \ldots & 0 & -\alpha \theta & 1+2 \alpha \theta
\end{array}\right] \\
-q=\left[\begin{array}{c}
(1+2 \alpha \theta) g_{1}^{m+1}-\alpha \theta g_{2}^{m+1}-b_{1}^{m} \\
g_{i}^{m+1}+\alpha(1-\theta)\left(g_{i-1}^{m+1}-2 g_{i}^{m+1}+g_{i+1}^{m+1}\right)-b_{i}^{m} \\
\vdots \\
-\alpha \theta g_{N-2}^{m+1}+(1+2 \alpha \theta) g_{N-1}^{m+1}-b_{N-1}^{m}
\end{array}\right] .
\end{gathered}
$$

Os elementos da diagonal de $A$ são positivos, os elementos por fora da diagonal são negativos, e a matriz é estritamente diagonal dominante. Isto 
é, $A$ é uma matriz de Minkowski (M-matriz). Esta propriedade garante a existencia e unicidade do problema de complementaridade $(q, A)[19]$.

\section{Algoritmo de Brennan e Schwartz}

Brennan e schwartz introduziram um algoritmo para o problema de precificar uma opção put americana em [32]. O algoritmo de solução é baseado na eliminação de Gauss onde a restrição de exercício adiantado da opção é manipulada em uma forma simples. A discretização no espaço e no tempo do problema de complementaridade (1.10) conduz a uma seqüência de problemas de complementaridade estacionaria. Daqui, em cada passo de tempo $m, m=0, \ldots, M$, o problema

$$
\left\{\begin{array}{l}
A u^{m+1} \geq b^{m} \\
u^{m+1} \geq g^{m+1} \\
\left(A u^{m+1}-b^{m}\right)^{T} \cdot\left(u^{m+1}-g^{m+1}\right)=0
\end{array}\right.
$$

Precisa ser resolvido. Aqui, a matriz $A$ tem origem na discretização da equação diferencial parcial. Mostramos como o algoritmo de Brennan e Schwartz é aplicado em cada passo de tempo para resolver o problema (2.8). Nós descrevemos o algoritmo para uma call americana, da seguinte forma: A eliminação transforma cada linha do sistema de equações lineares $A u=b$ da forma

$$
p_{j} u_{j}+s_{i} u_{j+1}=f_{j}
$$

para $j=1, \ldots, n$, onde os coeficientes são dados por

$$
\begin{array}{lcl}
p_{1}=A_{11}, & s_{1}=A_{12}, & f_{1}=b_{1} \\
p_{j}=A_{j j}-\frac{A_{i i-1}}{p_{i-1}} A_{i-1 i}, & s_{i}=A_{i i+1}, & f_{i}=b_{i}-\frac{A_{i i-1}}{p_{i-1}} f_{i-1}, \\
p_{n}=A_{n n}-\frac{A_{n n-1}}{p_{n-1}} A_{n-1 n} & \mathrm{e} & f_{n}=b_{n}-\frac{p_{n-1}}{A_{n n-1}} f_{n-1} .
\end{array}
$$


A solução $u$ do problema (2.8) é obtida usando as equações (2.9) e a função payoff da opção call. Deve-se começar resolvendo $u_{n}$ da equação (2.9) quando $j=n$; se $u_{n}$ é menor que o valor do payoff então iguala-se $u_{n}=g_{n}$. Após você prossegue resolvendo para $u_{n-1}$, etc.

\section{Algoritmo de Elliot e Ockendon}

O segundo algoritmo que apresentamos e devido a Elliot e Ockendon. Eles dão um algoritmo para problemas de complementaridade linear em [17] e este algoritmo directo é aplicado a problemas de precificação de opções americanas em [8]. Para começar, o problema de opção americana é transformado de maneira que a restrição de exercício adiantado seja igual à função zero ao invés de ser a função payoff. Esta transformação é feita usando as seguintes substituições para o problema (2.8):

$$
z^{j+1}=u^{j+1}-g^{j+1} \text { e } q^{j+1}=A g^{j+1}-b^{j}
$$

Com estas notações o problema de complementaridade pode-se formular como em Huang e Pang [16], na seguinte forma:

$$
\left\{\begin{array}{c}
A z+q \geq 0 \\
z \geq 0 \\
(A z+q)^{T} \cdot z=0
\end{array}\right.
$$

onde $j=0, \ldots, M$. Esta apresentação do problema de complementaridade também é considerada em [17] e [8]. Assumimos que a matriz de discretização $A$ é uma $M$-matriz. Ainda mais, assume-se que o vetor $q^{j+1}$ tem uma forma especifica, a qual é descrita na proposição 2.1.

No seguinte o método de solução de novo é considerado em um instante de tempo, portanto o índice do passo no tempo é omitido. $\mathrm{O}$ algoritmo 
de Elliot e Ockendon, para o problema de complementaridade linear (2.10), como referenciado em [17] é:

$$
\begin{aligned}
\text { inicializar } & i=1, \quad \alpha_{1}=A_{11}, \quad f_{1}=-q_{1} / A_{11} \\
\text { Passo : } & i \rightarrow i+1 \\
& \gamma_{i-1}=A_{i-1 i} / \alpha_{i-1} \\
& \alpha_{i}=A_{i i}-A_{i i-1} \gamma_{i-1} \\
& f_{i}=-\left(q_{i}+A_{i i-1} f_{i-1}\right) / \alpha_{i} \\
& \text { se } f_{i}>-q_{i+1} / A_{i+1 i} \quad \text { então volta ao passo } \\
\text { substituição regressiva } & k=i \\
& z_{k}=f_{k} \\
& i=k-1: 1:-1 \\
& z_{i}=f_{i}-\gamma_{i} z_{i+1}
\end{aligned}
$$

Observe que $\gamma_{i}$ e $\alpha_{i}$, são elementos da decomposição LU e $f_{i}$ é o vetor intermediário após a substituição directa. Este algoritmo deve ser empregado em cada passo de tempo e a solução do problema de precificação original é obtida mediante $u^{j+1}=z^{j+1}+g^{j+1}$.

\section{Análise do método}

Em cada instante de tempo a solução do problema de complementaridade linear satisfaz a equação diferencial parcial de Black e Scholes em uma região e na outra região é igual à restrição de exercício adiantado. Portanto, a cada passo de tempo $j$ existe um no $k_{j}$ na discretização o qual divide o eixo $x \mathrm{em}$ duas regiões separadas.

Mostramos que a solução satisfaz a equação diferencial parcial nos nos onde o valor intermediário depois da substituição directa é positivo e a solução é igual à restrição de exercício adiantado (aqui a função zero) nos nos onde o valor intermediário depois da substituição directa é negativo. 
Inicialmente ${ }^{2}$, assumimos que a solução tem uma forma especifica. Em forma precisa, supomos que a solução $z^{j}$ tema a forma

$$
z_{i}^{j}>0, \quad i=0, \ldots, k_{j}, \quad \text { e } z_{i}^{j}=0, \quad i=k_{j}+1, \ldots, N-1
$$

Estudamos o algoritmo de Elliot e Ockendon e especificamente a condição no laço do passo o qual pode ser reformulado com uso das seguintes relações de equivalência:

$$
\begin{aligned}
& f_{i+1}=-\left(q_{i+1}+A_{i+1 i} f_{i}\right) / \alpha_{i+1}>0 \\
& \Leftrightarrow-\left(q_{i+1}+A_{i+1 i} f_{i}\right)>0 \\
& \Leftrightarrow q_{i+1}<-A_{i+1 i} f_{i} \\
& \Leftrightarrow-q_{i+1} / A_{i+1 i}<f_{i}
\end{aligned}
$$

onde $A_{i+1 i}$ é negativo pela propriedade $M$-matriz de $A$. Também os $\alpha_{i}$ são positivos por causa desta propriedade. Assumimos que $k_{j}$ é o menor índice para o qual $f_{k_{j}+1}<0$. Em concordância com o algoritmo de Elliot e Ockendon e a equivalência acima, temos que $z_{i+1}=0$ para $i=k_{j}, \ldots, n-1 \mathrm{e}$ ainda mais, o que resta da solução pode ser calculada usando substituição regressiva a qual da em $z_{i}=f_{i}-\gamma_{i} z_{i+1}$ para $i=1, \ldots, k_{j}$.

Nós podemos ver que o algoritmo de Elliot e Ockendon é mais eficiente que o algoritmo de Brennan e Schuwartz no sentido que o segundo calcula todas as componentes do vetor solução $z$. Por sua vez o algoritmo de Elliot e Ockendon calcula só um número $k$, com $k<(N-1)$, de componentes do vetor $z$, aquelas entradas que são diferentes de zero. o qual carrega um tempo menor e sem custo computacional adicional.

Na seguinte proposição, assume-se que $A$ é uma matriz tridiagonal, que

\footnotetext{
${ }^{2}$ Este fato será provado na próxima proposição.
} 
denotaremos da seguinte forma:

$$
A=\left[\begin{array}{ccccc}
a_{1} & c_{1} & 0 & \ldots & 0 \\
b_{2} & a_{2} & c_{2} & \ldots & 0 \\
0 & \ddots & \ddots & \ddots & 0 \\
\vdots & \ddots & b_{n-1} & a_{n-1} & c_{n-1} \\
0 & \ldots & 0 & b_{n} & a_{n}
\end{array}\right]
$$

Proposição 2.1 - (i) Se a matriz A satisfaz as mesmas condições que em (2.6), ou seja, os elementos da diagonal de A são positivos, os elementos por fora da diagonal são negativos, e a matriz é estritamente diagonal dominante então existe uma única solução de (2.10).

- (ii) Se $q_{i}<0, i=1,2, \ldots, k_{0}$, e $q_{i} \geq 0, i=k_{0}+1, \ldots, n-1$, então existe um $k>k_{0}$ tal que $z_{i}=0$, para $i>k$ e $z_{i}>0$ para $i \leq k$.

\section{Prova}

- (i)A matriz $A$ é uma matriz de Minkowski irredutivel, portanto o resultado e garantido por um teorema que pode ser consultado na referência [33].

- (ii)Se $q_{i}<0$, então $z_{i} \geq-\left(q_{i}+c_{i} z_{i+1}+b_{i} z_{i-1}\right) / a_{i}>0$, a ultima desigualdade se verifica dado que $a_{i},-b_{i}$ e $-c_{i}$ são positivos. Dai $z_{i}>0$ para todo $i<k_{0}$. Seja $(k+1)$ o primeiro inteiro maior que $k_{0}$ para o qual $z_{k}=0$, assim o PCL é satisfeito por $z_{i}=0 ; w_{i}=q_{i} ; i>k$.

$\triangleright$

A proposição anterior mostra uma forma de tomar vantagem do nosso problema particular: suponha que a solução do PCL (2.10) de fato satisfaz $z_{i}>0$ para $i \leq k$; e $z_{i}=0$ para $i>k$, deste modo para se achar $z$ deve-se resolver 
o sistema

$$
\left.\begin{array}{c}
a_{1} z_{1}+c_{1} z_{2}+q_{1}=0 \\
b_{i} z_{i-1}+a_{i} z_{i}+c_{i} z_{i+1}+q_{i}=0 \quad 1=2, \ldots, k-1 \\
b_{k} z_{k-1}+a_{k} z_{k}+q_{k}=0
\end{array}\right\}
$$

Nós observamos que aplicando eliminação gaussiana directa para (2.11), a primeira componente encontrada é $z_{k}$; se além $z_{k}$ também satisfaz $b_{k+1} z_{k}+$ $q_{k+1} \geq 0$, então o índice $k$ tem sido achado e só resta a substituição regressiva para determinar a única solução do PCL. Este modo de proceder é numericamente estável dado que $A$ é diagonal dominante. O conteo de operações é $(5 k+4)+(k-1)$ multiplicações e divisões, o último termo provem de testar a desigualdade para $f_{i}$. Em [65] o número de operações para o método aplicado ao mesmo problema é $k^{2}+3 k$; e $(5 k-4)+\frac{3}{2}\left(k^{2}-k_{0}^{2}\right)+\frac{3}{2}\left(k-k_{0}\right)$ para o método em [34]

Nas aplicações, $k-k_{0}=O(1 / h)$ e geralmente $k>k_{0}$ [17]. Também, dado que $k=O(1 / h)$, reduzindo na metade $h$ dobra o número de operações para o algoritmo.

Só resta mostrar que o vetor $q$ tem o sinal necessário para a aplicação da proposição 2.1. A cada passo de tempo, o vetor $q$ no PCL (2.8) provem da substituição $q=A g^{m+1}-b^{m}$, onde $A$ é dado em (2.6), o vetor $b$ em (2.5) e o vetor resultante $q$ é dado pela fórmula (2.7). Vamos supor o caso de uma call americana para a função payoff $g$.

Supondo uma discretização do operador de difusão pelo método $\theta \mathrm{com}$ 
$\theta=1$, obtemos o seguinte esquema de diferenças finitas

$$
\begin{gathered}
(1+2 \alpha) u_{n}^{m+1}-\alpha\left(u_{n-1}^{m+1}+u_{n+1}^{m+1}\right) \geq u_{n}^{m} \\
u_{n}^{m+1} \geq g_{n}^{m+1} \\
u_{0}^{m+1}=g_{0}^{m+1}, \quad u_{N}^{m+1}=g_{N}^{m+1}, \quad u_{n}^{0}=g_{n}^{0}
\end{gathered}
$$

ou equivalentemente com uso de notação matricial obtemos o problema de complementaridade

$$
\begin{gathered}
A u^{m+1} \geq u^{m} \\
u^{m+1} \geq g^{m+1} \\
\left(A u^{m+1}-u^{m}\right)\left(u^{m+1}-g^{m+1}\right)=0
\end{gathered}
$$

onde $A$ é a matriz tridiagonal com entradas $A=\left[\begin{array}{lll}-\alpha & (1+2 \alpha) & -\alpha\end{array}\right]$.

Proposição 2.2 O vetor $q$ satisfaz o seguinte padrão de sinal

$$
\begin{aligned}
& q_{i}<0 \quad x_{i} \in\left[x_{\text {inf }}, x_{s}\right) \\
& q_{i} \geq 0 \quad x_{i} \in\left(0, x_{s}\right) \\
& q_{i} \geq 0 \quad x_{i} \in\left[x_{s}, x_{s u p}\right]
\end{aligned}
$$

\section{Prova}

Na secção 1.1 obtivemos uma expressão para a função payoff na forma

$$
g(x, \tau)= \begin{cases}e^{\beta \tau} e^{\alpha x}\left(e^{x}-1\right) & (x>0) \\ 0 & x \leq 0\end{cases}
$$

onde

$$
\alpha=-\frac{1}{2}\left(k_{2}-1\right), \quad \beta=-\frac{1}{4}\left(\left(k_{2}-1\right)^{2}+4 k_{1}\right) .
$$

Daí obtemos que

$$
\begin{aligned}
g_{n}^{m+1} & =e^{\beta \Delta \tau} g_{n}^{m} \\
& =e^{\delta} g_{n}^{m}, \quad \delta=\beta \Delta \tau
\end{aligned}
$$


- Seja $x_{i} \in\left[x_{\text {inf }}, 0\right]$.

Nesse caso $g_{i}^{m+1}=0$ e portanto

$$
\begin{aligned}
q_{i}^{m+1} & =-b_{i}^{m}=-u_{i}^{m} \\
& <0 .
\end{aligned}
$$

- Seja $x_{i} \in\left(0, x_{s}\right)$ o vetor $q$ é dado pela expressão

$$
\begin{aligned}
q & =\left(A g^{n+1}\right)_{n}-u_{n}^{m} \\
q & =\left(A g^{n+1}\right)_{n}-g_{n}^{m}-\left(u_{n}^{m}+g_{n}^{m}\right) \\
e^{-\alpha x_{n}} q_{n} & =e^{-\alpha x_{n}}\left(A g^{n+1}\right)_{n}-g_{n}^{m}-\left(u_{n}^{m}+g_{n}^{m}\right) \\
& \approx \Delta \tau e^{-\alpha x_{n}}\left(g_{\tau}-g_{x x}\right)-e^{-\alpha x_{n}}\left(u_{n}^{m}+g_{n}^{m}\right)
\end{aligned}
$$

Agora

$$
\begin{array}{r}
g_{\tau}-g_{x x}=e^{\beta \tau} e^{\alpha x} \frac{2 D}{\sigma^{2}}\left\{e^{x}-\frac{r}{D}\right\} \\
e^{-\alpha x_{n}} g_{\tau}-g_{x x}=e^{\beta \tau} \frac{2 D}{\sigma^{2}}\left\{e^{x}-\frac{r}{D}\right\}
\end{array}
$$

ou seja a função $e^{-\alpha x_{n}} g_{\tau}-g_{x x}$ é crescente em $x$. De outro lado

$$
\begin{aligned}
u(x, \tau) & =e^{\beta \tau} e^{\alpha x} V\left(K e^{x}, \cdot\right) \frac{1}{K} \\
e^{-\alpha x_{n}} u(x, \tau) & =e^{\beta \tau} V\left(K e^{x}, \cdot\right) \frac{1}{K} \\
e^{-\alpha x_{n}} g(x, \tau) & =e^{\beta \tau}\left(e^{x}-1\right) \quad \text { portanto } \\
e^{-\alpha x_{n}}(u-g) & =e^{\beta \tau}\left(V\left(K e^{x}, \cdot\right) \frac{1}{K}-\left(e^{x}-1\right)\right)
\end{aligned}
$$

derivando com respecto à variavel $x$ temos

$$
\begin{aligned}
{\left[e^{-\alpha x_{n}}(u-g)\right]_{x} } & =e^{\beta \tau}\left(e^{x} V_{s}-e^{x}\right) \\
& =-e^{\beta \tau} e^{x}\left(1-V_{s}\right)<0
\end{aligned}
$$

pois $0<V_{s}<1$, este fato pode ser consultado na referência [38]. Assim a função $e^{-\alpha x_{n}}(u-g)$ é decrescente, daí a função $-e^{-\alpha x_{n}}(u-g)$ é crescente. 
Da análise anterior concluimos que

$$
e^{-\alpha x_{n+1}} q_{n+1}>e^{-\alpha x_{n}} q_{n}
$$

Portanto se $q_{m} \geq 0$ então $q_{m+1} \geq 0$ e isto prova que a partir do primeiro índice $i$ para qual $q_{i} \geq 0$ se cumpre que $q_{n} \geq 0$ para todo $n>i$.

- Seja $x_{i} \in\left[x_{s}, x_{\text {sup }}\right]$

Nesse caso temos $u_{i}^{m+1}=g_{i}^{m+1}$ e assim o vetor $q$ toma a forma seguinte $q_{i}=\left(A g^{m+1}-b\right)_{i}$ o em equivalentemente

$$
\begin{aligned}
& g_{n}^{m+1}-\alpha\left(g_{n-1}^{m+1}-2 g_{n}^{m+1}+g_{n+1}^{m+1}\right)=g_{n}^{m} \\
& g_{n}^{m+1}-\alpha(\Delta x)^{2}\left(g_{x x}\right)_{n}^{m+1}=g_{n}^{m} \\
& g_{n}^{m+1}-g_{n}^{m}=\alpha(\Delta x)^{2}\left(g_{x x}\right)_{n}^{m+1} \\
& \frac{g_{n}^{m+1}-g_{n}^{m}}{\Delta \tau}=\left(g_{x x}\right)_{n}^{m+1} \\
& \left(g_{\tau}\right)_{n}^{m}+O(\Delta \tau)=\left(g_{x x}\right)_{n}^{m}+O\left((\Delta x)^{2}\right) \\
& \left(g_{\tau}-g_{x x}\right)_{n}^{m}>0 .
\end{aligned}
$$

Estudo da função $g$ para a última desigualdade:

Nós usamos o seguinte resultado sob opções americanas

$$
\begin{array}{lll}
g_{\tau}-g_{x x}<0 & \text { se } & x<\ln \left(\frac{r}{D}\right) . \\
g_{\tau}-g_{x x}>0 & \text { se } & x>\ln \left(\frac{r}{D}\right) .
\end{array}
$$

Este fato pode comprovar se observamos que

$$
g_{\tau}-g_{x x}=e^{\beta \tau} e^{(\alpha+1) x} \frac{2}{\sigma^{2}}\left\{D e^{x}-r\right\}
$$

Queremos saber o comportamento de $g_{\tau}-g_{x x}$. Para o caso de uma call 


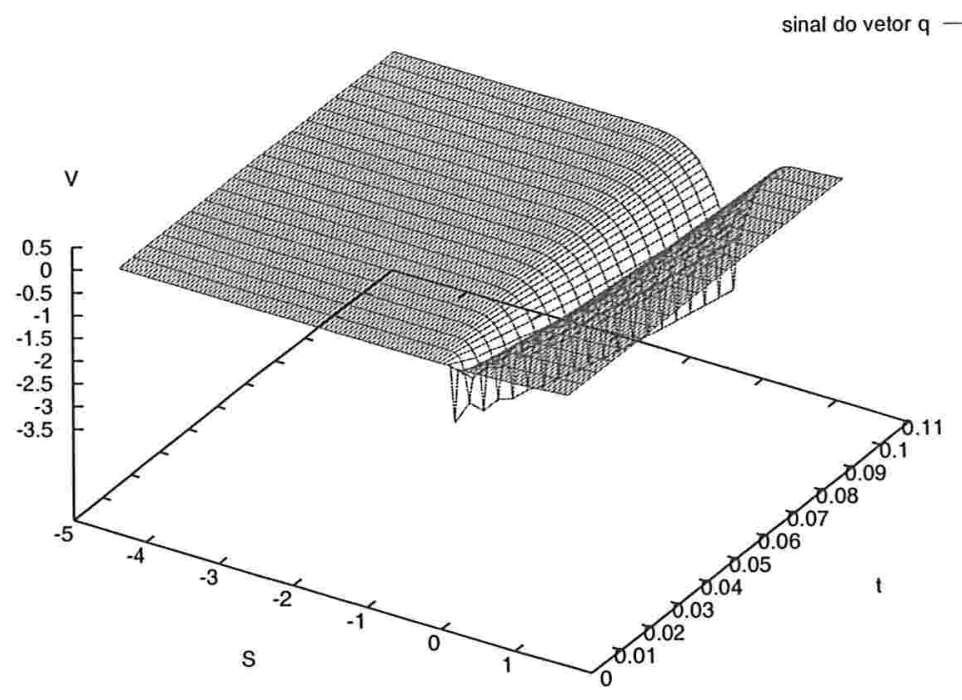

Figura 2.2: Gráfico da superficie gerada pelo padrão de sinal do vetor $q$ para uma opção call de estilo americano do teste 5 calculada pelo esquema de Crandall-Douglas, com $\alpha=1$.

temos o seguinte

$$
\begin{gathered}
g_{\tau}-g_{x x}=A(\tau) e^{\frac{1}{2} k_{2} x} \frac{2}{\sigma^{2}}\left\{D e^{\frac{1}{2} x}-r e^{-\frac{1}{2} x}\right\} \\
g_{\tau}-g_{x x}>0 \Leftrightarrow D e^{\frac{x}{2}}>r e^{-\frac{x}{2}}
\end{gathered}
$$

Mas $S=K e^{x}$ e portanto

$$
g_{\tau}-g_{x x}>0 \quad \Leftrightarrow \quad S>K \frac{r}{D}
$$

Pela teoria de opções americanas $S(\tau)$ é uma função não decrescente sendo que $S(0)=\max \left\{\frac{r}{D} K, K\right\}$. Isto implica que

$$
S(\tau) \geq S(0) \quad \tau \geq 0
$$




$$
\begin{gathered}
S(\tau) \geq \max \left\{\frac{r}{D} K, K\right\} \\
\text { se } \tau>0 \text { então } e^{x(\tau)}>\frac{r}{D} \quad \text { o que significa que } \\
\text { para } x>x_{\tau} \text { se cumpre que } g_{\tau}-g_{x x}>0
\end{gathered}
$$

$\triangleright$

Teorema 1 Se $u_{n}^{m}$ é solução do problema de complementaridade (2.12) então Para $m \geq 1$ se cumpre que $u_{n}^{m} \geq e^{\delta} u_{n}^{m-1}$.

\section{Prova}

Vamos razonar por inducção. Seja $m=1$ então temos que o PCL (2.12) se transforma em

$$
\begin{gathered}
A u^{1} \geq u^{0} \\
u^{1} \geq g^{1} \\
\left(A u^{1}-u^{0}\right)\left(u^{1}-g^{1}\right)=0
\end{gathered}
$$

onde as condições de fronteira que se cumprem são $u^{0}=g^{0} \mathrm{e}$

$$
u^{1} \geq g^{1}=e^{\delta} g^{0}=e^{\delta} u^{0}
$$

Isto prova o teorema para o primeiro instante de tempo, caso $m=1$.

\section{Hipotóse de inducção}

Supondo que o enunciado é valido para todo $p$ com $p \leq m$ queremos provar que o resultado é valido para $p=m+1$. Temos o PCL

$$
\begin{gathered}
A u_{n}^{m+1} \geq u_{n}^{m} \\
u_{n}^{m+1} \geq g_{n}^{m+1} \\
\left(A u_{n}^{m+1}-u_{n}^{m}\right)\left(u_{n}^{m+1}-g_{n}^{m+1}\right)=0
\end{gathered}
$$


Note que como $u_{n}^{m} \geq e^{\delta} u_{n}^{m-1}$ temos que

$$
\left\{\begin{array}{l}
A u_{n}^{m+1} \geq e^{\delta} u_{n}^{m-1} \\
u_{n}^{m+1} \geq g_{n}^{m+1}
\end{array}\right.
$$

Agora $e^{\delta} u_{n}^{m}$ é solução de

$$
\left\{\begin{array}{l}
A\left(e^{\delta} u_{n}^{m}\right) \geq e^{\delta} u_{n}^{m-1} \\
e^{\delta} u_{n}^{m} \geq e^{\delta} g_{n}^{m}=g^{m+1}
\end{array}\right.
$$

Das equações (2.14) e (2.15) concluimos

$$
u_{n}^{m+1} \geq e^{\delta} u_{n}^{m}
$$

Isto prova o teorema para o caso geral.

$\triangleright$

Uma consequiência imediata é que como $u_{n}^{m+1} \geq e^{\delta} u_{n}^{m}$ e $e^{\delta} u_{n}^{m}=e^{\delta} g_{n}^{m} \geq$ $g_{n}^{m+1}$ temos que a fronteira se move para a direita.

Observação Uma prova similar pode ser dada para o padrão de sinal do vetor $q$ quando aplicamos os esquemas de diferenças finitas de CrandallDouglas, Crank-Nicolson e a fórmula BDF2 ao operador de difusão. Essencialmente a prova faz uso dos seguintes fatos: a estrutura da matriz $A$, consistência dos esquemas de diferenças finitas com a equação diferencial parcial e as propriedades da função $g$.

Para opções americanas de venda, o vetor de complementaridade tem o padrão de sinal $q_{n} \geq 0$ para $x_{i n f}<x<x_{s}$ e $q_{n}<0$ para $x_{s}<x<x_{\text {sup }}$. Assim, basta iverter siplesmente a ordem das componentes dos vetores $q$ e $z$, do mesmo modo fazer com os elementos da diagonal principal, sub-diagonal e super-diagonal da matriz $A$, intercambiando a sub-diagonal com a superdiagonal, e proceder com o algoritmo como descrito acima. 


\section{Capítulo 3}

\section{Testes computacionais}

A implementação apresentada nesta seção foi desenvolvida na linguagem de programação GNU ${ }^{1}$ FORTRAN 90 utilizando precisão simples e executado num computador do IME-USP com as seguintes características: processador AMD² Opteron 242, 512Mb. de memória RAM e sistema operacional Linux, distribuição Debian ${ }^{3}$.

\section{Teste 3}

Nós apresentamos agora simulações numéricas para ilustrar a aproximação à formulação de Black e Scholes ilustrada acima. Para este exemplo, foram utilizados os seguintes valores dos parâmetros $r=0.1, \sigma=0.4, k=1$, $D=0.07, x_{\text {inf }}=-4.6, x_{\text {sup }}=1.6, N=124, \Delta \tau=0.005$. Portanto, $\Delta x=0.05, \alpha=2$.

A Tabela 3.1 apresenta os resultados para uma put americana após 20 iterações $(\tau=0.1)$. E na Figura 3.1 é mostrada a solução aproximada pelo esquema Crandall Douglas e utilizando o método de Elliott Ockendon para

\footnotetext{
$1_{\text {www.gnu.org }}$

${ }^{2}$ www.amd.com

${ }^{3}$ www.debian.org
} 
os cálculos dos PCL's.

\begin{tabular}{|l|l|l|l|l|}
\hline Preço do Ativo & Explicito & Crank-Nicolson & BDF & Douglas1 \\
\hline 0.57 & 0.4230501 & 0.4230501 & 0.4230501 & 0.4230501 \\
0.60 & 0.3945534 & 0.3945404 & 0.3945523 & 0.3945473 \\
1.0 & 0.1508205 & 0.1507020 & 0.1508193 & 0.1507912 \\
1.4 & $4.3224 E-2$ & $4.3173 E-2$ & $4.3203 E-2$ & $4.3191 E-2$ \\
2.0 & $1.1787 E-2$ & $1.1805 E-2$ & $1.1795 E-2$ & $1.1766 E-2$ \\
2.5 & $3.0555 E-3$ & $3.0868 E-3$ & $3.0868 E-3$ & $3.0489 E-3$ \\
3.0 & $1.2009 E-3$ & $1.2263 E-3$ & $1.2319 E-3$ & $1.1997 E-3$ \\
3.4 & $4.2817 E-4$ & $4.4472 E-4$ & $4.5128 E-4$ & $4.2923 E-4$ \\
4.0 & $1.3515 E-4$ & $1.4366 E-4$ & $1.4813 E-4$ & $1.3636 E-4$ \\
4.7 & $2.4236 E-5$ & $2.6263 E-5$ & $2.7459 E-5$ & $2.4608 E-5$ \\
\hline
\end{tabular}

Tabela 3.1: Teste $3, \Delta \tau=0.005$

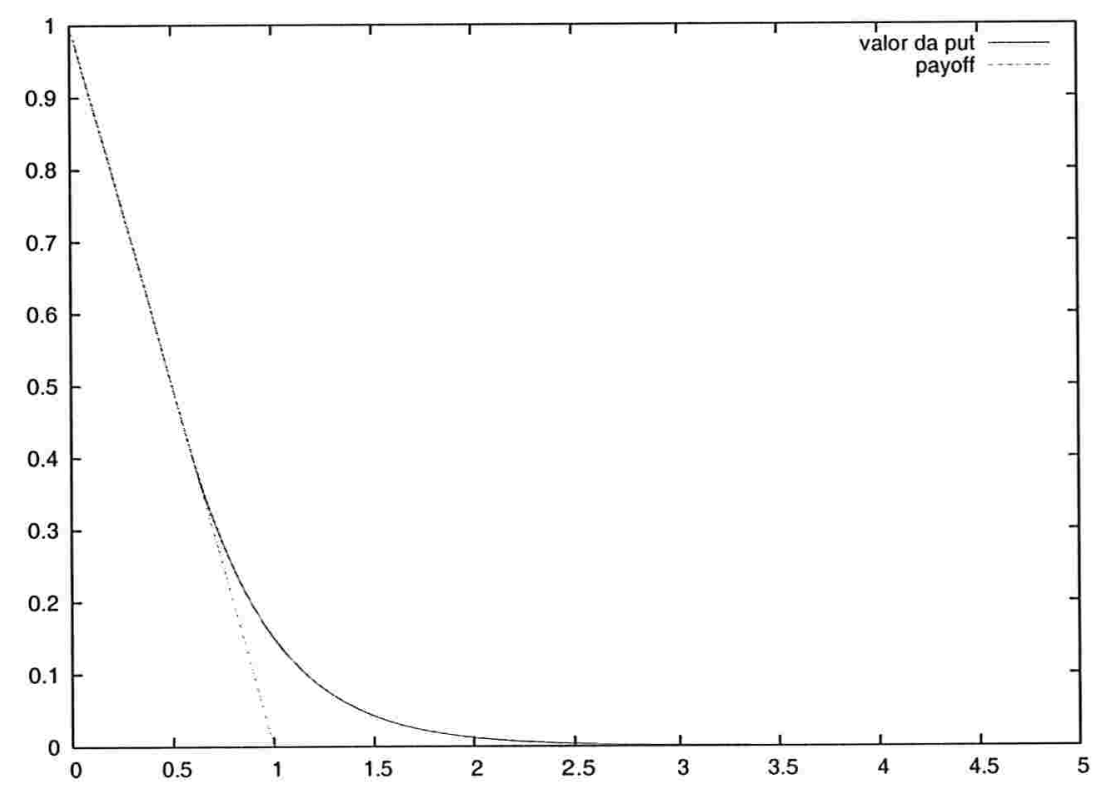

Figura 3.1: Gráfico em $t=0.005$ do preço de uma opção put de estilo americano do teste 3 calculada pelo esquema de Crandall-Douglas, $\operatorname{com} \alpha=2$ e $\Delta x=0.05$. 


\section{Teste 4}

Considere uma opção call americana a seis meses com uma taxa de dividendo $D=0.03$. O preço de exercício é 100 , a taxa de juros livre de risco é $r=0.03$, e a volatilidade é $40 \%$ ao ano. A fronteira direita é fixada em $x_{\text {sup }}=1.6$ e a esquerda em $x_{\text {inf }}=-4.6$. Um tamanho de passo no tempo $M=496$ com $\alpha=0.16$ é tomado para todos os métodos. Como os dados da solução exata não são conhecidos utilizamos o método éxplicito com um refinamento de malha tal que a convegência do método é alcançada. Para propósitos de comparação nós tomamos esses valores como sendo os valores exatos da opção. Os resultados são apresentados na Tabela 3.2.

\begin{tabular}{|l|l|l|l|l|}
\hline Preço do Ativo & Valor Verdadeiro & Crank-Nicolson & BDF & Douglas1 \\
\hline 40.15 & 0.002942 & 0.002965 & 0.002966 & 0.002936 \\
50.28 & 0.048605 & 0.048705 & 0.048706 & 0.048542 \\
60.65 & 0.333538 & 0.333569 & 0.333565 & 0.333258 \\
70.46 & 1.207727 & 1.207241 & 1.207235 & 1.207059 \\
80.85 & 3.265165 & 3.263724 & 3.263726 & 3.264007 \\
90.48 & 6.525598 & 6.523326 & 6.523339 & 6.524074 \\
100 & 11.10845 & 11.10576 & 11.10577 & 11.10674 \\
110.51 & 17.61820 & 17.61559 & 17.61561 & 17.61651 \\
120.62 & 25.05209 & 25.04994 & 25.04994 & 25.05058 \\
\hline
\end{tabular}

Tabela 3.2: Teste 4, $\tau=0.04$ valor de uma call americana com vencimento em 6 meses, o número de passos de tempo foi $256, \alpha=0.16$.

\subsection{Teste de Convergência}

Neste experimento numérico comparamos as taxas de convergência dos métodos Crank-Nicolson, a fórmula BDF2 e o esquema de Crandall-Douglas. Os problemas de complemetaridade linear a cada passo de tempo foram resolvido s pelo algoritmo de Elliott e Ockendon. Para os cálculos de uma 
opção americana de compra tomamos os seguintes valores dos parâmetros $\sigma=0.6, r=0.25, K=10, D=0.2, x_{\text {inf }}=-6.9, x_{\text {sup }}=1.9$ e $T=1$. Os resultados são apresentados na Tabela 3.3.

Para o caso dos esquema Crank-Nicolson e BDF2 e Crandall-douglas a solução numérica apresenta presição similar para os tamanhos de passo espacial $N=176,352,704$, Da Tabela 3.3 vemos que quando o número de passos espaciais crece de 704 para 1408 a solução numérica obtida pelo esquema de Crandall-Douglas tem maior precisão que os outos métodos avaliados.

\begin{tabular}{|l|l|l|l|l|}
\hline N Espaco & M Tempo & Crank-Nicolson & BDF & Douglas1 \\
\hline 176 & 216 & $1.565918 E-3$ & $1.558269 E-3$ & $1.243767 E-3$ \\
352 & 432 & $3.744659 E-4$ & $3.728284 E-4$ & $2.875099 E-4$ \\
704 & 864 & $8.023125 E-5$ & $8.061523 E-5$ & $7.961523 E-5$ \\
1408 & 1728 & $6.196267 E-6$ & $6.120635 E-6$ & $3.635415 E-8$ \\
\hline
\end{tabular}

Tabela 3.3: Teste 5, Erro máximo e taxa de convergência da solução numérica de uma call americana com vencimento em 12 meses.

\subsection{O delta}

Neste último experimento numérico, estudamos a influência do esquema de discretização temporal para a função delta, a qual é a derivada do preço da opção com respecto a $S$. Nas figuras 3.2 e 3.3, mostramos as funções delta para opções call e put americanas nos casos do método de Crank-Nicolson e o esquema de BDF2.

Como pode ser visto o esquema BDF2 suavisa as funções delta enquanto oscilações indesejaveis surgem do uso do método de Crank-Nicolson. Estas oscilações são localizadas perto do preço de exercício $K$, onde a derivada espacial da condição inicial é descontínua. Os valores dos parâmetros são os mesmos que os usados no teste de convergência e foi usada $N=2048 \mathrm{e}$ 
$M=32$. O tamanho do passo no tempo $M$ foi relativamente pequeno com a finalidade de mostar as oscilações.

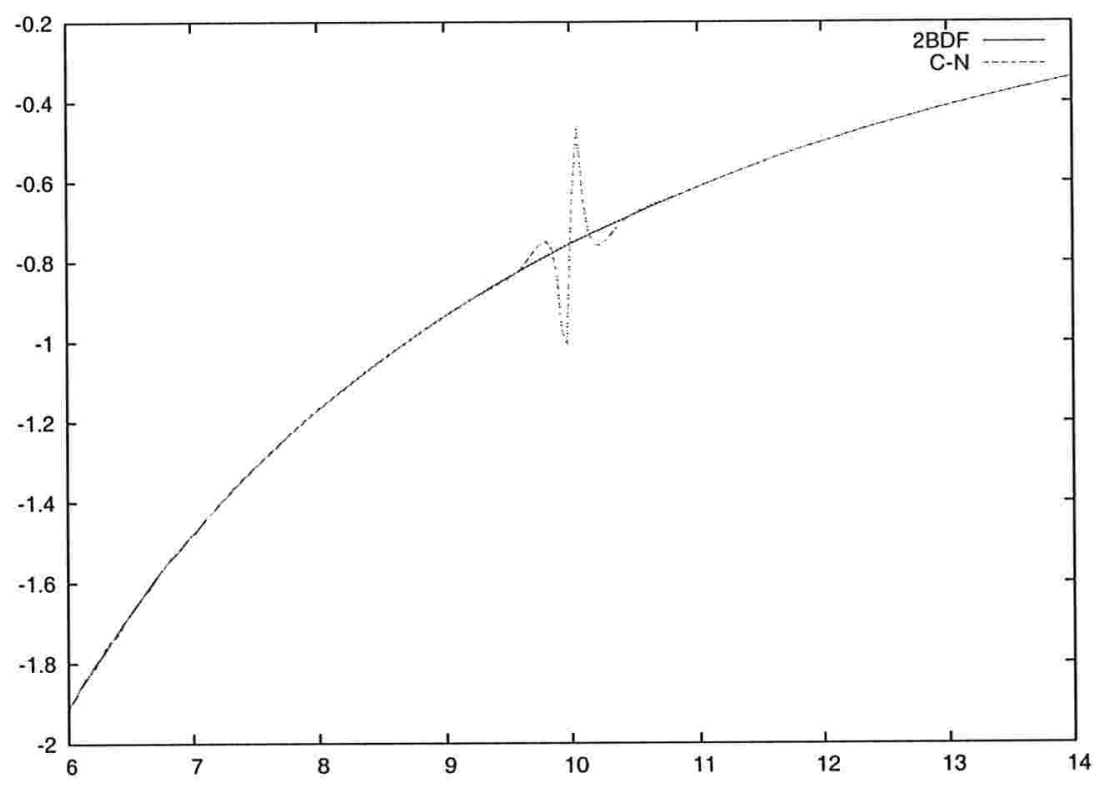

Figura 3.2: Gráfico em $T=1$ da função delta de uma opção put americana do teste 4 calculada pelo esquema de Crank Nicolson e por 2BDF, com $\Delta x=$ 0.004 .

Nós ainda refinamos mais a malha para uma grade de 8188 pontos espaciais por 128 pontos temporais e observamos da figura 3.4 que o esquema de Crank-Nicolson continua apresentado oscilações perto do preço de exercício.

Com isto comprovamos que mantendo $\alpha$ constante o esquema de CrankNicolson não é dissipativo. Além disso, dado que a função payoff $g$ tem derivada discontínua para o instante inicial, o esquema de Crank-Nicolson acaba apresentado dados errados da solução numérica da função delta.

Testes para o cálculo da delta foram realizados para o esquema de CrandallDouglas e observamos que este apresenta oscilasões na solução numérica, portanto devemos utilizar métodos dissipativos que suavisem essas oscilasões, para este fim nós fazemos uso o da fórmula BDF2. 


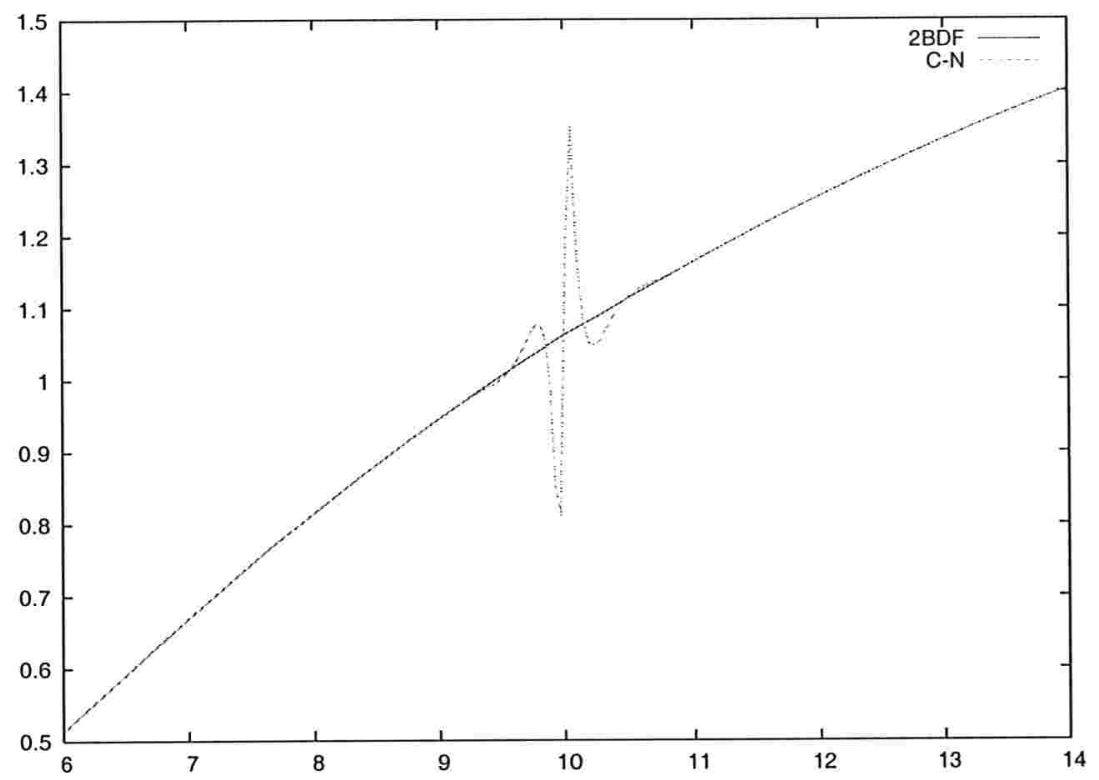

Figura 3.3: Gráfico em $T=1$ da função delta de uma opção call americana do teste 4 calculada pelo esquema de Crank Nicolson e 2BDF, com $\Delta x=0.004$.

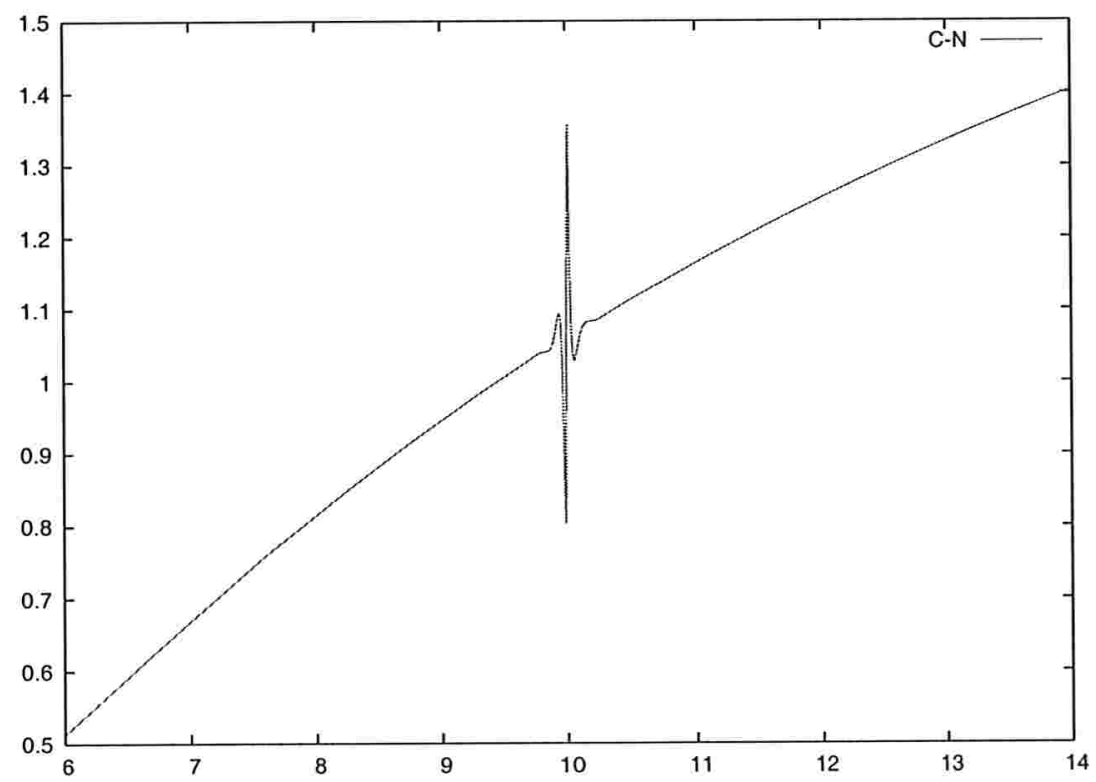

Figura 3.4: Gráfico em $T=1$ da delta de uma opção call americana do teste 4 calculada pelo esquema de Crank Nicolson $\Delta x=0.001$. 


\section{Capítulo 4}

\section{Conclusões}

Neste trabalho, aplicamos métodos de diferenças finitas para precificar op̧ões vanilla de estilo americano. Foi investigado esquemas de discretizações no tempo e no espaço e se utilizou um método direto para o problema de complementaridade linear.

Aplicamos o método $\theta$ para a discretização do operador de difusão. Este esquema pode chegar a ser de quarta ordem de precisão no espaço e incondicionalmente estável como no caso $\theta=\frac{1}{2}-\frac{1}{12 \alpha}$. Nós estudamos a influência da discretização no tempo para o calculo da grega delta da opção e aplicamos a fórmula BDF2 para a discretização no tempo e verificou-se nos experimentos numéricos que esta possui precisão similar ao método incondicionalmente estável de Crank Nicolson, além disso, comprovamos que a fórmula BDF2 produz menores oscilasões que o método de Crank Nicolson.

Quando aplicamos às desigualdades variacionais para opções americanas $\mathrm{o}$ as discretizações levam a problemas de complementaridade $(A, \mathrm{q})$ linear que precisam ser resolvidos em cada passo do tempo, onde a matriz $A$ é uma Mmatriz tridiagonal e o vetor $q$ tem as propriedades de sinais necessárias para a aplicação do algoritmo de Elliott Ockendon como observado anteriormente na secção 2.2. Nesta formulação a restrição de exercício adiantado da opção 
é manipulada usando as propriedades do sinal do vetor $q$. 


\section{Apêndice}

O presente capítulo começa com a derivação da equação de precificação para opções européias baseada na construção de um portfolio cujo valor siga os fluxos de dinheiro do derivativo em função do tempo. Há outras aproximações fundamentais para derivar as equações de precificação para derivativos européios, uma delas faz uso do teorema de Feynman-Kac ( [12] secção 3.3), que estabelece que a esperança condicional de um processo estocástico obedece uma equação diferencial parcial. Existe ainda uma aproximação mais moderna para o problema de precificação, chamado 'replication principle' vide [12].

\section{Precificação de Opções pela Aproximação Di- ferencial}

No seu famoso trabalho, Black e Scholes transformaram o problema de precificação na terefa de resolver uma equação diferencial parcial parábolica com uma condição final. Do lado do uso de equações diferenciais parciais como uma ferramenta técnica, a principal idéia conceptual de Black e Scholes radica na construcção de um portfolio livre de risco consistindo de posições tomadas sobre bonds, opções, e o ativo objeto. Considerações para este modelo 
- O preço do ativo objeto segue o movimento geométrico browniano $d S=$ $\mu S d t+\sigma S d W \operatorname{com} \mu, \sigma$ constantes;

- não há dividendos durante a vida do derivativo;

- a negociação com títulos é contínua;

- é permitido a venda a descoberto de títulos, com a total utilização dos recursos;

- não há custos de transação;

- todos os títulos são infinitamente divisíveis;

- não há oportunidades de arbitragem livre de risco;

- a taxa de juro livre de risco de curto prazo, $r$, é constante e igual para todos os vencimentos.

Construcção de um portfolio livre de risco e o problema de Cauchy. Em seu artigo ([11]), Black e Scholes consideram um portfolio cosistindo de posições variáveis em um bond ${ }^{1} S_{0}$ e um ativo objeto $S$, e exatamente de uma opção vendida. Por simplicidade vamos supor o caso de uma call européia com preço de exercício $K$. Black e Scholes supõem que o preço da call no tempo $t$ pode ser representado como uma função $C(S(t), t)$ do tempo e do preço atual $S(t)$. Naturalmente, o preço da opção na maturidade $T$ é dado por

$$
C(S(T), T)=\left(S_{T}-K\right)^{+}
$$

Para continuar com o argumento precisamos da seguinte definição:

Portfolio $\left(\phi_{0}, \phi_{1}\right)$ É um par $\phi_{0}$ e $\phi_{1}$ o qual descreve respectivamente o número de unidades de ativos e de bonds que temos no tempo $t$.

\footnotetext{
${ }^{1} \mathrm{O}$ modelo do preço de um bond é $S_{0}(t)=I e^{r t}, S_{0}(0)=: I, I \in \mathbb{R}^{+}$.
} 
Apêndice

Estratégia Com a idéia de portfolio vem a idéia de estratégia. A descrição $\left(\phi_{0}(t), \phi_{1}(t)\right)$ é uma estratégia dinâmica que detalha a quantidade de cada componente a segurar em cada instante. Um portfolio é de auto financiamento se a mudança em seu valor depender somente da mudança do preço do ativo.

A idéia principal de Black e Scholes é seguir uma estratégia de negocio (trading) de autofinanciamento $\left(\psi_{0}(t), \psi_{1}(t)\right)$ em ativos e bonds tais que o processo da riqueza incluindo exatamente uma opção call vendida

$$
\Pi(t):=\psi_{0}(t) S_{0}(t)+\psi_{1}(t) S(t)-C(S(t), t)
$$

não possui nenhuma flutuação aleatoria. Este é considerado como um portfolio livre de risco. Se então o preço de opção satisfaz as condições necessárias para a aplicação da fórmula de Ito, i.e., se $C(S, t)$ for suficientemente suave, então obtemos:

$$
\begin{aligned}
d \Pi(t) & =\psi_{0}(t) d S_{0}(t)+\psi_{1}(t) d S(t)-d C(S(t), t) \\
& =\left[\psi_{0}(t) S_{0}(t) r+\psi_{1}(t) S(t) \mu\right. \\
& \left.-\left(C_{t}+C_{S} S \mu+\frac{1}{2} C_{S S} S^{2}(t) \sigma^{2}\right)\right] d t \\
& +\left(\psi_{1}(t) S(t) \sigma-C_{S} S(t) \sigma\right) d W(t)
\end{aligned}
$$

Para $\Pi(t)$ ser o processo da riqueza correspondente ao portfolio livre de risco os coeficientes de difusão devem-se anular (para eliminar a incerteça). Daqui devemos ter

$$
\psi_{1}(t)=C_{S}(S, t)
$$


Apêndice

Por razões de arbitragem ${ }^{2}$, o processo da riqueza $\Pi(t)$ deve comportar-se como um múltiplo do preço do bond, nós assim requeremos

$$
d \Pi(t)=r \Pi(t) d t
$$

Substituindo agora $\psi_{1}(t)$ por $C_{S}(S, t)$, o requeremento acima sobre o coeficiente drift implica

$$
\begin{aligned}
r \Pi(t) & =r \psi_{0}(t) S_{0}(t)+\mu C_{S} S-C_{t}+C_{S} S \mu+\frac{1}{2} C_{S S} S^{2}(t) \sigma^{2} \\
& =r\left(\psi_{0}(t) S_{0}(t)+\psi_{1}(t) S(t)-C(S(t), t)\right)+r C(S(t), t) \\
& +(\mu-r) C_{S} S(t)-\left(C_{t}+\mu C_{S} S(t)+\frac{1}{2} C_{S S} \sigma^{2} S(t)^{2}\right) \\
& =r \Pi(t)+\underbrace{\left(r C-r C_{S} S(t)-C_{t}-\frac{1}{2} C_{S S} \sigma^{2} S(t)\right)}_{=0}
\end{aligned}
$$

Junto com a condição final em $t=T$ e algumas condições apropriadas de regularidade, nós obtemos assim que o preço da call $C(S(t), t)$ resolve o seguinte problema de Cauchy

$$
\left\{\begin{array}{l}
\frac{1}{2} \sigma^{2} S(t)^{2} C_{S S}+(r-D) S C_{S}+C_{t}-r C=0, \quad(S, t) \in(0, \infty) \times[0, T) \\
C(S, T)=(S-K)^{+}, \quad S \in(0, \infty) \\
\operatorname{com} \\
C \in C((0, \infty) \times[0, T]) \cap C^{1,2}((0, \infty) \times[0, T))
\end{array}\right.
$$

Onde temos acrescentado o termo devido à taxa de dividendos.

\footnotetext{
${ }^{2} \mathrm{~A}$ equação para $d \Pi$ não involucra $d W_{t}$, o portfolio é livre de risco durante um periodo de tempo $d t$ e portanto ganha a taxa instantânea de retorno $r$, ou seja $d \Pi=r \Pi d t$.
} 
Apêndice

\section{Observação}

O processo seguido pelo preço de uma ação sem dividendos pode ser escrito como

$$
d S=\mu S d t+\sigma S d z
$$

ou

$$
\frac{d S}{S}=\mu d t+\sigma d z
$$

Esse desenvolvimento do comportamento dos preços de uma ação é às vezes conhecido como movimento browniano geometrico. A partir do lema de Itô, o processo seguido por uma função $f$, de $S$ y $t$ é:

$$
d f=\left(\frac{\partial f}{\partial S} \mu S+\frac{\partial f}{\partial t}+\frac{1}{2} \frac{\partial^{2} f}{\partial S^{2}} \sigma^{2} S^{2}\right) d t+\frac{\partial f}{\partial S} \sigma S d z
$$

Para um instrumento americano, a aplicação do principio de não arbitragem reduz o problema de precificação para a cálculo do máximo, sobre todas as estratégias de exercício, de uma esperança sobre o espaço dos fatores subjacentes que determinam o preço do instrumento.

Sob apropriadas condições, o cálculo destas esperanças se reduz à solução de um problema de complementaridade diferencial parcial [10]. 


\section{Referências Bibliográficas}

[1] S. Karamardian. The Complementarity Problem. Math. Programming 2(1972)107- - 129 .

[2] Complementarity and Fixed Point Problems. Volume 7 of Mathematical Programming Study

[3] Diaz Tinoco., Hernandez Trillo. Futuros y Opciones Financieras: Una Introduccion. 3a. ed. Mexico: Limusa 2005.

[4] Samuli Ikonen., Jari Toivanen. Pricing American Options Using LU Decomposition. Reports of the Department of Mathematical Information Technology. Series B. Scientific Computing. No. B4/2004

[5] Rose-Anne Dana., Monique Jeanblanc. Financial Markets in Continuous Time. Springer Finance. Textbook.2002

[6] Domingo Tavella., Curt Randall. Pricing Financial Instruments-The Finite Difference Method. Wiley. 2000.

[7] Paul Wilmott., Sam Howison, Jeff Dewynne. The Mathematics of Financial Derivatives-A student Introduction. Cambrige. 1997.

[8] Brian J., McCartin., Suzanne M. Labadie. Accurate and Efficient Pricing of Vanilla Stock Options Via the Crandall-Douglas Scheme. Applied Mathematics and Computation 143 (2003) 39-60. 
Referências bibliográficas

[9] Ritchmeyer R., Morton K. Difference Methods for Initial-Value Problems. New York: Interscience. 1967.

[10] Jaillet P., Lamberton D. Lapeyre Variational Inequality and the Pricing of American Options. Acta applicandae Mathematicae, 21, (1990), 263289.

[11] F. Black, M. Scholes The Pricing of Options and Corporate Liabilities. Journal of Political Economics 81, (1973), 637-659.

[12] Ralf Korn, Elke Korn. OPtion Pricing and Portfolio OptimizationModern Methods of Financial Mathematics. Graduate Studies in Mathematics, volume 31. American Mathematical Society.

[13] William Shaw. Advanced Finite Difference Schemes. Presentation on behalf of Oxford Centre for Computational Finance, Mathematical Institute. Oxford University.

[14] Shameer Sukha. Advanced Mathematics of Finance Honours Project: Finite Difference Methods for Pricing the American Put Option. December $10,2001$.

[15] Pauly Oliver. Numerical Simulation of American Option. Universitat Ulm. Facultàt für Mathematik und Wirtschaftswissenschaften, am 5. Mai 2004.

[16] Jacqueline Huang, Jong-Shi Pang. Option Pricing and Linear Complementarity. Journal of Computational Finance, vol2 no 3, 1998.

[17] C.M. Elliot, J.R Ockendon. Weak and Variational Methods for Moving Boundary Problems. Pitman, 1982, pp. 115 - 117.

[18] John C. Strikwerda. Finite Difference Schemes and Partial Differential Equations. University of Wisconsin - Madison, 1989. 
Referências bibliográficas

[19] G. Windisch. M-matrices in Numerical Analisys. vol 115, BSB B. G. Teubner Verlagsgesellschaft, Leipzig, 1989.

[20] O. Axelson. Iterative Solution Methods. Cambridge University Press, Cambridge 1996, pp. 200-213.

[21] D. Kinderlehrer G. Stimpacchia. An Introduction to Variational Inequalities and their Applications. Academic Press 1980.

[22] H. Samelson R. M. Thrall O. Wesler. A partition theorem for Euclidean n-space. Proc. Amer. Math. Soc. 9, 1958, 805-807.).

[23] R. W. Cottle, A. F. Veinott Jr.Polyhedral sets having a least element. Math. Programming 3, 1972, 238 - 249.)

[24] R. Glowinski, J.-L. Lions, R. Trémollières. Analyse numérique des inéquations variationnelles. Dunod, Paris, 1976.

[25] A. Friedman. Partial Differential Equations of Parabolic Type. Krieger Publishing, New York, 1983.

[26] J. F. Harper. Reducing Parabolic Partial Differential Equations to Canonical Form. European Journal of Applied Mathematics 5 (Part 2), June 1994, $159-164$.

[27] K. W. Morton, D. F. Mayers.Numerical Solution of Partial Differential Equations. Second Edition. Cambridges. 2005.

[28] J. Crank. Free and Moving Boundary Problems. Oxford University Press. Oxford 1984.

[29] A. Freidman. Variational Principles and Free Boundary Problems Krieger Publishing, New York, 1988. 
Referências bibliográficas

[30] R. W. Cottle, J. S. Pang. The Linear Complementarity Problem Academic Press, New York. 1992.

[31] J. C. Hull. Options, Futures, and other Derivativos Prentice-Hall, 3 ed.,1997.

[32] M.J.Brennan, E.S.Schwartz.The valuation of American put options Journal of Finance, 32 (1977), pp. 449 - 462.

[33] Cottle, R.W. and Sacher, R.S. On the Solution of Large structured Linear Complementarity Problems: the Tridiagonal Case. App. Math. Optim. 3 (1977), 321-340

[34] Sacher, R.S. On the Solution of Large Structured LinearComplementarity Problems II. Tech. Rep. 73-5, Stanford Univ. Dep. Operations Research, 1973.

[35] P. Wilmott, J. Dewynne, and S. Howison. Option Pricing: Mathematical Models and computation. Oxford Financial Press. Oxford, 1993.

[36] P. A. Forsyth and K. R. Vetzal. Quadratic convergence for valuing American Options using a penalty method. SIAM Journal on Scientific Computing, 23 (1993), pp. 2095-2122.

[37] S. Ikonen and J. Toivanen. Operator Splitting methods for American Option Pricing. Applied Mathematical Letters. To appear.

[38] M. A. H. Dempster and J. P. Hutton. Fast numerical valuation of American, exotic and complex options. Applied Mathematical Finance, 4 (1997), pp. 1-20. 